DOE/ER/53296--3

DE93 000885

Technical Progress Report

\title{
Application of Optical Fluctuation Diagnostics to Transport Studies in High Temperature Tokamak Plasmas
}

\author{
Professor Raymond J. Fonck \\ Principal Investigator \\ Department of Nuclear Engineering and Engineering Physics \\ University of Wisconsin-Madison
}

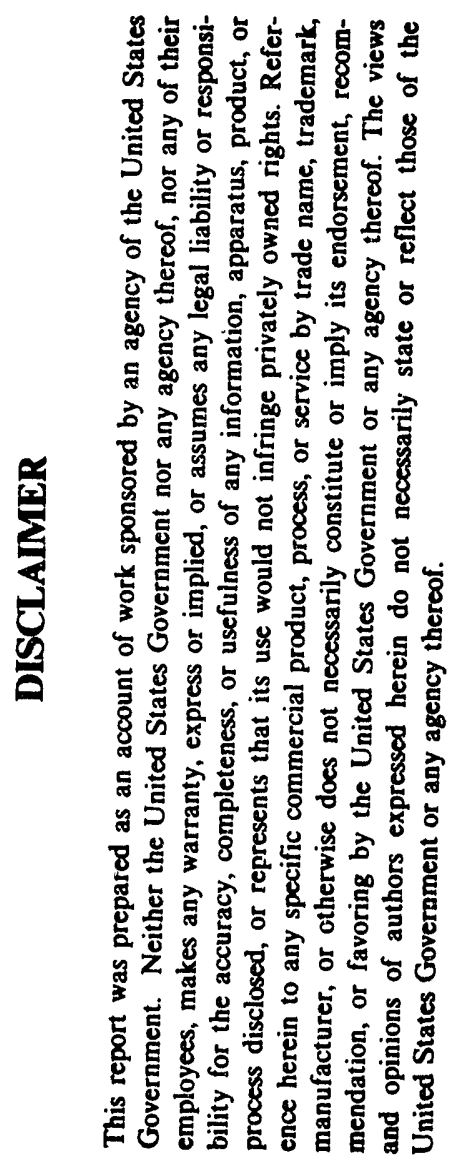

\author{
U.S. Department of Energy \\ Grant No. DE-FG02-89ER53296
}

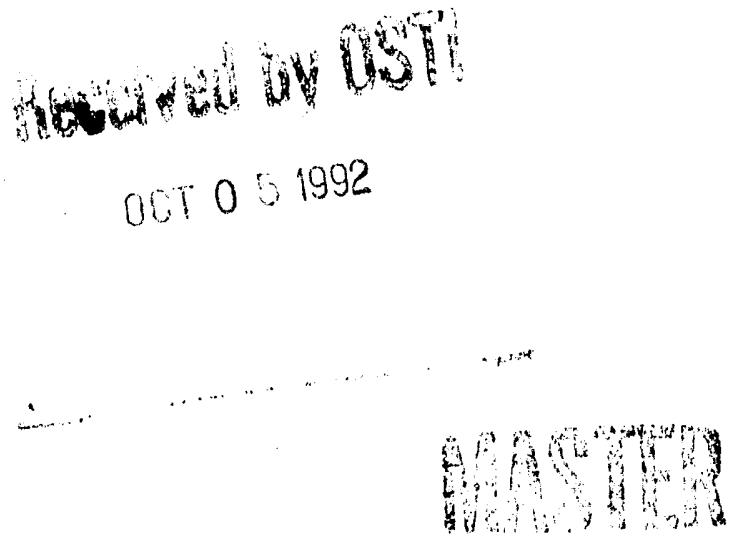




\section{Technical Progress Report for GRANT NO. DE-FG02-89ER53296}

\section{Review nf Work on Beam Emission Spectroscopy (1990-1992)}

\section{A. Overview of BES}

Beam Emission Spectroscopy (BES) is an optical technique for studying plasma density fluctuations. 1,2 The fluctuations in the collisionally excited fluorescence from an injected neutral beam (Fig. 1) are, to first order, directly proportional to the local fluctuations in plasma density. The beam fluorescence is spectrally distinguished from background plasma emission either by its Doppler shift or by injecting a species different than the majority ion species (e.g., a He neutral beam into a D plasma). ${ }^{2}$

BES has several advantages as a fluctuation diagnostic:

- It is nonperturbative.

- It is applicable both to the plasma edge and to the hotter core region of large tokamaks.

- It is sensitive to long wavelength fluctuations $\left(k_{\perp}=0-2 \mathrm{~cm}^{-1}\right.$ or higher).

- BES has both radial and poloidal spatial resolution.

- The physics of collisionally excited fluorescence, while nontrivial, is well understood and thus, to first order, there are no significant difficulties in data interpretation.

- It is relatively simple to implement a multichannel system. This allows full, multipoint correlation analysis which avoids the ambiguities inherent in the two-point correlation techniques often employed in fluctuation experiments.

Balanced against these advantages are two key limitations:

- The irreducible statistical fluctuations in the detected photon flux are convolved with the fluctuations induced by the plasma, setting a lower limit to the detectable fluctuation level and also necessitating fairly long integration times.

- Fluctuations in the neutral beam density can be misinterpreted as local plasma fluctuations. In particular, strong plasma density fluctuations may induce a fluctuation in the neutral beam density (by varying the local beam attenuation coefficient). 
Our initial grant period covered all the University of Wisconsin activities in a joint UW-PPPL collaboration to develop and apply BES to study long-wavelength density fluctuations in TFTR. This chapter summarizes the main results of our efforts to establish BES as a reliable diagnostic approach and results of measurements of turbulent density fluctuations on TFTR.

The BES development effort over the past two and a half years has concentrated on exploiting the advantages described above to study plasma density fluctuations in TFTR while attempting to understand and ameliorate the limitations of the technique. The effect of photon statistics is reduced by maximizing the number of detected photons. This requires high-étendue optics and high quantum efficiency $(\mathrm{QE})$ detectors. During the initial grant period, we developed new photodiode detectors with an order of magnitude greater sensitivity than the photomultiplier tubes originally employed. 3

There is no known technique for eliminating plasma-induced fluctuations in the neutral beam density and thus effort has focussed on signal processing techniques which allow the local plasma density fluctuations to be distinguished from fluctuations in the neutral beam density. 4

In addition to these performance-motivated developments there has been considerable effort devoted to issues of remote operation. Developments in both hardware and software have made it possible for a remote operator at the University of Wisconsin to successfully operate the BES diagnostic and acquire data during several experiments. 5 While peripheral to the central effort, these developments provide valuable experience and tools for future remote collaborations.

\section{B. Hardware Installation and Development}

The early phase of the BES development activity at UW involved prototype testing of a simple 4channel system on the PBX-M tokamak while working with the TFTR team to design a more capable, 16-channel system for TFTR. This system and all support facilities (ports, data acquisition, etc.) were fabricated by PPPL staff and installed in early 1990. Since that time several upgrades have been performed, including the development and installation of two successive generations of improved detectors, a dedicated, high-speed RISC workstation for data analysis, an automatic fiber optic switcher, and software to allow remote operation. In addition, a 2-channel BES test bed using a low power diagnostic beam was installed and operated on the Phaedrus-T tokamak at UW.

The TFTR BES system images one of the TFTR heating neutral beams (beam line 4, Fig. 2) onto an array of fiber optic bundles which carry the image to the set of detectors. ${ }^{6}$ The imaging optics consist of a reentrant, vacuum mirror (which is enclosed in a stainless steel, protective heat shield), a 
$20 \mathrm{~cm}$ diameter viewing port and a high-throughput collection lens (Fig. 3). The heat shield box protects the vacuum mirror from the plasma heat flux and minimizes the deposition of carbon and other substances on the first surface mirror. The shield box is shuttered to completely isolate the mirror from the plasma when BES is not operating. The collecting lens is a three-element compound lens with a 20 $\mathrm{cm}$ diameter and a focal length of $34 \mathrm{~cm}$. It is designed to maximize light collection and to utilize the full numerical aperture $(f / 2)$ of the fiber optics.

The fiber optic arrav consists of a 55 bundle radial array and five, 10 bundle vertical (poloidal) arrays (Fig. 4). Each fiber optic bundle consists of four 1 -mm core fused silica fibers in a $2 \times 2$ pattern. Scans in both the radial and vertical (i.e., poloidal) directions are available by choice of the available uptical fiber views. To scan the field of view in the plasma, the fiber bundle connections are switched. Originally, this was done by manually connecting the detectors with the desired fiber bundles. However, this technique was extremely labor intensive and prone to error. In 1991 an automated fiberswitching apparatus was designed and installed. 7 This allows the position of the radial and poloidal arrays to be easily and rapidly scanned on a shot-to-shot basis and also facilitates remote operation of the system. Any combination of radial and poloidal channels is possible (e.g., 20 radial channels).

The detector apparatus consists of an interference filter and a set of relay lenses to focus the image of the fiber bundle onto the detector. The interference filter is located between the relay lenses and is tuned to admit the Doppler shifted $D_{\alpha}$ emission from the neutral beam while rejecting the unshifted $D_{\alpha}$ from the plasma periphery.

In the original BES prototype red-enhanced, Gallium Arsenide photomultiplier tubes (PMT) were used. Subsequently, two successive generations of detectors have been developed at UW. In 1991 the PMT detectors were replaced by avalanche photodiodes (APD). In late 1991 the APD detectors were replaced by photoconductive photodiodes (PPD). The PPD detectors have a quantum efficiency near 0.7 at $\mathrm{D}_{\alpha}$ and give a factor of four improvement in signal-to-noise ratio over the APD's and a factor of 12 improvement over the PMTs (Fig. 5). ${ }^{3}$ The photodiodes selected (EG\&G Reticon FFD-100) have a very low dark (thermal) current which allows the detector circuit to operate at room temperature without unacceptable thermal noise. ${ }^{3}$ Using these detectors BES is able to resolve broadband density fluctuations down to the $0.1 \%$ level, which is in excess of the original design specifications for the TFTR BES system.

The system has been configured to facilitate remote operation. The control of most of the BES subsystems (e.g., shutter, data acquisition, fiber scanning) is accomplished via software which allows the diagnostic to be controlled from the remote site (University of Wisconsin). In addition, software has been developed which allows remote user access to much of the same information that is available in the TFTR control room. For example, the machine operations and the physics run logs are now 
implemented as remotely accessible computer databases (rather than the former practice of using handwritten overhead projections). This has allowed the BES diagnostic to be operated successfully under the sole control of a remote operator at the University of Wisconsin during several of the experimental runs in late 1991 and early 1992.

In addition to the main effort on TFTR, a smaller two-channel BES system has been installed on Phaedrus-T. This system has been used primarily as a test bed in support of TFTR BES development. For example, the initial tests of the ultra-low noise PPD detectors and diagnostic comparisons between BES and Langmuir probes were performed on Phaedrus-T. ${ }^{8}$

\section{Diagnostic Physics and Analysis Techniques}

\section{Atomic Physics Excitation Model}

BES relates measurements of the local neutral beam fluorescence to the local density fluctuations. That is, for given beam and plasma parameters

$$
\tilde{n}_{\text {rms }} / n=c\left(n_{e}, T_{e}, Z_{\text {eff }}, E_{\text {beam }}, L_{c}\right) \tilde{I}_{r m s} / I
$$

where $\tilde{n}_{r m s} / n$ is the normalized, rms density fluctuation amplitude, $\tilde{I}_{r m s} / I$ is the normalized, rms amplitude of the beam fluorescence and $\mathrm{c}$ is a proportionality factor which depends on the local electron temperature and density, the effective ionic charge $\left(\mathrm{Z}_{\mathrm{eff}}\right)$, the neutral beam energy, $\mathrm{E}_{\text {beam, }}$, and the correlation length of the fluctuations, $L_{c}$.

The evaluation of the proportionality factor factor, $c$, requires an atomic model of the collisional excitation and radiative processes including ionization and collisional deexcitation. In the early stages of this work a multistep, steady-state, homogenous plasma model due to Boley and Janev was employed. We collaborated with J. Callen's plasma theory group at UW to develop a more sophisticated time-dependent code which allows realistic plasma temperature and density profiles to be included. $9 \mathrm{~A}$ time-dependent treatment is important because the time for the beam neutrals to traverse a density fluctuation is not, in general, long enough for the excited states to establish a new equilibrium. This implies that the ratio of $\left(\tilde{\mathrm{n}}_{\mathrm{rms}} / \mathrm{n}\right)\left(\tilde{I}_{\mathrm{rms}} / \mathrm{I}\right)$ depends weakly on the the scale length of the density fluctuations. Figure 6 shows the intensity fluctuations which would be induced by density fluctuations of $1 \mathrm{~cm}$ and $5 \mathrm{~cm}$ width. In the case of the $1 \mathrm{~cm}$ fluctuation the excited states do not reach a new, saturated equilibrium while in the case of the $5 \mathrm{~cm}$ fluctuation saturation does occur. 


\section{Spectral Analysis Techniques}

In the course of the BES system development on TFTR an extensive suite of signal processing techniques including auto- and cross-spectral analysis (autopower, cross-phase and coherence) and time-delay correlation analysis have been integrated into the TFTR BES data acquisition and archival structure. 4 Auto- and cross-spectral analyses are well established in studies of plasma fluctuations. Time-delay correlation analysis is less frequently employed as it is most powerful when applied to multipoint data (most measurements of plasma fluctuations have tended to be two-point rather than multipoint). Correlation analysis of multipoint data allows the group propagation velocity, $v_{g}$, the correlation length, $L_{c}$, the decorrelation time, $\tau_{c}$, and the spatial autopower spectrum (k-spectrum), $S(k)$, to be derived. Each of these quantities can be directly related to present theoretical models.

Figure 7 shows an example of the correlation coefficient for 3 poloidal channels. The correlation coefficient is defined as 13

$$
\rho(\Delta x, \Delta t)=\frac{\langle\tilde{I}(x, t) \tilde{I}(x+\Delta x, t+\Delta t)\rangle}{\sqrt{\left.\left\langle\tilde{I}(x, t)^{2}\right) \tilde{I}(x+\Delta x, t+\Delta t)^{2}\right\rangle}}
$$

where $\tilde{I}$ is the fluctuating part of the measured signal, i.e., $I(t)=I_{D C}+\tilde{I}(t), x$ represents a spatial coordinate (either radial or poloidal) and the angular brackets indicate an ensemble average (which, in practice, is evaluated as an average in time with the assumption that the data are ergodic) and $\Delta t$ is the time lag between the two channels. 10 In Fig. 7, the overall shape of the correlation coefficient for each channel is similar but shifted in time indicating a poloidal propagation. If the time delay at which the correlation coefficient peaks is plotted versus the poloidal channel separation, $\Delta z$, the group velocity of the fluctuations is given by the slope (Fig. 8).

A measure of the spatial scale of the fluctuations is given by the correlation length, $L_{c}$, defined as the distance in which the envelope of the correlation function decreases to $1 / e$ times it value at $\Delta x=0$, $\Delta \mathrm{t}=0$.

Figure 9 shows a poloidal correlation envelope profile from a representative TFTR shot $(\mathrm{r} / \mathrm{a}=0.73)$. The superimposed exponential fit gives a correlation length of $2.4 \mathrm{~cm}$, which is typical for TFTR. Note that the use of an exponential fit is arbitrary but seems to be a reasonable approximation to the data (except near $\Delta z=0$ where finite spatial resolution effects are important). The radial correlation envelope profile and its associated correlation length are found to be very similar. To date no large asymmetries between radial and poloidal correlation lengths have been observed.

A characteristic temporal scale is given by the decorrelation time, which is the time taken for the 
peak of the correlation envelope profile to decay to $1 / \mathrm{e}$ times its value at $\Delta x=0, \Delta t=0$. Figure 10 shows the peak value of the correlation envelope profile for each channel, plotted versus the delay at which it occurs. The superimposed exponential fit indicates a decorrelation time of $36 \mathrm{msec}$.

Information on the distribution of power among spatial scales in the turbulent fluctuations is given by the $k$-spectrum, which is defined as the Fourier transform of the correlation function at $\Delta t=0$. In general, for a system with finite spatial resolution, $S(k)$ is convolved with the instrumental spatiul response function. In order to obtain the true $\mathrm{k}$-spectrum it is necessary to deconvolve the instrumental effects.

The spatial response function for BES depends on the geometry of the intersection between the neutral beam and the optical lines of sight as well as the cross sectional density profile of the neutral beam. Techniques for deconvolving these spatial resolution effects are currently under development and will be aggressively pursued in the proposed grant period. However, k-spectra for long wavelength MHD modes (for which spatial resolution effects are not important) have been calculated. For example, see Fig. 25 below.

\section{Treatment of Systematic Effects}

Equation 1, which states that the local beam fluorescence is proportional to the local density fluctuations is, in practice, only an approximation. There are several systematic effects which must be accounted for in calculating the correlation functions. In particular, power supply ripple in the neutral beam density, the intrinsic photon statistical fluctuations, and modulations in the neutral beam density induced by the strong edge fluctuations all cause fluctuations in the observed beam fluorescence which are not related to the local plasma density fluctuations.

Power supply ripple is, in principle, avoidable. However, the heating neutral beams on TFTR were not designed for diagnostic use and are thus not optimized in this regard. Photon statistical intensity fluctuations and edge-induced beam density fluctuations are inherent to the BES technique and cannot be suppressed. Thus, signal processing techniques were developed to distinguish these systematic effects from the locally induced fluctuations. In this section these techniques are briefly discussed.

\section{3a. Photon Statistical Noise}

Photon statistical fluctuations, or photon noise, in the BES signal are a consequence of the quantum nature of light photons or equivalently, of the electrons in the detector photocurrent. This adds a Gaussian (i.e., frequency independent) component to the measured autopower spectrum which 
dictates the minimum detectable fluctuation level. Plasma fluctuations much smaller than the photon noise level are not distinguishable. A fundamental design goal of the BES system has been the minimization of the photon statistical noise by the use of high-throughput optics and development of very high quantum efficiency detectors. Simultaneously, amplifier noise must be minimized so that the total detector noise is dominated by photon statistics. Such photon-noise-limited operation is routinely achieved. The present TFTR BES system can distinguish broadband fluctuations down to the $0.1 \%$ level. Coherent modes, because of their narrower bandwidth, are easier to distinguish than the broadband plasma fluctuations and can be distinguished at even smaller levels.

Figure 11 shows a typical BES autopower spectrum from a standard L-mode discharge. The significant plasma fluctuations lie below about $100 \mathrm{kHz}$ while above this frequency the spectrum is essentially flat which indicates that photon noise is dominant. In general, the RMS photon noise level, $\tilde{N}$, may be estimated by $\tilde{N} \approx S\left(f_{\text {photon }}\right) B$ where $S\left(f_{\text {photon }}\right)$ is the autopower in some spectral region where photon noise is dominant and $\mathrm{B}$ is the detector bandwidth.

This measured noise level can then be used to correct the correlation profiles for the effects of photon noise. The correlation coefficient becomes

$$
\rho(\Delta x, \Delta t)=\frac{[\tilde{I}(x, t) \tilde{I}(x+\Delta x, t+\Delta t))-\delta(\Delta x) \tilde{N}(x)^{2}}{\sqrt{\left.\left(\left\langle\tilde{I}(x, t)^{2}\right)-\tilde{N}(x)^{2}\right)\left(\tilde{I}(x+\Delta x, t+\Delta t)^{2}\right)-\tilde{N}(x+\Delta x)^{2}\right)}}
$$

where $\delta(\Delta x)$ is the Kronecker delta function. Note that if the photon noise power dominates then this equation is ill-conditioned and small errors in the estimate of $\tilde{N}$ will cause large errors in $r$. In fact, we have found that small systematic uncertainties in the photon noise power (due to small drifts in the amplifier noise) tend to be the practical limit to our ability to detect and correlate small intensity fluctuations. Thus, one of the proposed developments in this grant period involves the development and application of "correlation-detectors" (discussed in the next chapter) to greatly reduce the systematic uncertainties in the photon noise, and hence enhance the effective detection sensitivity of the system.

\section{3b. Neutral Beam Power Supply Ripple}

Power supply ripple in the neutral beam density causes fluctuations in the beam fluorescence which are not proportional to the local plasma density fluctuations. However, this noise occurs at a set of discrete harmonics' of the power supply frequencies (120 and $720 \mathrm{~Hz}$ ). Thus, a comb filter at these 
harmonic frequencies can be used to remove this noise from the measureci signals. Such filtration is now routinely applied to all data. This ripple noise is most important for $\mathrm{f}<30 \mathrm{kHz}$ and often dominates the power spectrum below $5 \mathrm{kHz}$.

\section{3c. Edge-Induced Beam Density Fluctuations}

Any density fluctuation will tend to produce a modulation of the neutral beam density (through changes in the local attenuation coefficient). Because of this effect, the fluctuations in fluorescence from a given point in the plasma will not only reflect the local plasma density fluctuations but also the fluctuations in the plasma density upstream (i.e., outside) from the point of measurement. Both simulations ${ }^{9}$ and experiment ${ }^{4}$ indicate that this is, in fact, a real and significant effect which must be accounted for in the analysis of BES data (though the effect is more or less significant depending on the discharge parameters). The strongest contribution to this effect is from the strong fluctuations $\left(\tilde{n}_{\mathrm{rms}} / \mathrm{n} \approx 10-20 \%\right)$ which are typically seen at the edge and thus this non-local component of the signal is referred to as 'edge-induced' beam density fluctuations. 9 Because this interfering signal can distort the determination of both $\tilde{n}_{\mathrm{rms}} / \mathrm{n}$ and correlation functions, and it has often been cited by the fusion community as the greatest source of concern in interpreting BES (and Heavy Ion Beam Probe) measurements as local plasma fluctuations, we discuss our treatrinent of these edge-induced commonmode signals in some detail here.

Edge-induced fluctuations can be recognized by two hallmark features: 180 degree phase shifts with respect to the edge and very long radial correlation lengths. 9 Edge-induced beam density fluctuations are 180 degrees out of phase with the edge fluctuations which cause them because a positive density fluctuation at the edge will increase the collisional excitation of the neutral beam and thus cause a positive local intensity fluctuation. It also increases the beam attenuation which leads to a negative fluctuation in the fluorescence at all points further along the beam. This also explains the very long radial correlation lengths associated with this effect.

Since this effect gives rise to very long correlation lengths which could be misinterpreted as intrinsic plasma properties, it has been singled out as a diagnostic issue of particular concern (similar to concerns for the HIBP). Thus, we have spent considerable effort during the past year both to understand the effects of edge-induced beam density fluctuations on local BES measurements and to develop analysis techniques to minimize or eliminate them.

The time-dependent, multi-step atomic model discussed above has been used to simulate this effect. 9 Figure 12 shows the predicted magnitude of the edge-induced fluctuations for various edge fluctuation levels and fluctuation widths. Given that the experimentally measured radial correlation length at the edge is on the order of $2 \mathrm{~cm}$ these results imply that the edge should induce fluctuations of 
the fluorescent intensity in the core on the order of $0.1-0.2 \%$. This is in reasonable agreement with experimental measurements (see below). These simulations confirm the experimental observation that discharges with broad density profiles and strong edge fluctuations (which are characteristic of L-mode discharges) are more prone to edge-induced beam density fluctuations than are discharges with more peaked density profiles (such as supershots).

Since edge-induced beam density fluctuations are generally present they must be corrected during the data analysis. The fact that beam density fluctuations give rise to very long radial correlation lengths suggests an approach to distinguish between the intensity fluctuations induced by local density fluctuations and those which are edge-induced.

In general the measured fluctuation signal, $\tilde{I}(x, t)$, will consist of both local and non-local (beam density fluctuation) components

$$
\tilde{i}\left(r_{1}, t\right)=\tilde{I}_{\text {plasma }}\left(r_{1}, t\right)+\tilde{I}_{\text {beam }}\left(r_{1}, t\right)
$$

where the subscript 'beam' refers to the non-local beam density flustuations induced by all the plasma density fluctuations upstream (i.e., outside) of the measurement point at $r_{1}$. Because the dominant contributions to this term are from the large plasma density fluctuations at the edge $(r / a \approx 1)$, these fluctuations are referred to as 'edge-induced'. However, the technique described here does not depend on the beam density fluctuations being induced only at the edge and is readily generalized to include beam density fluctuations induced throughout the plasma. 4 Assuming that the local density fluctuations and the edge fluctuations are uncorrelated (which is the case if the density fluctuations are due to plasma turbulence) then the local RMS intensity fluctuation amplitude, $\tilde{\mathrm{I}}_{\mathrm{rms}}$, is given by

$$
i_{\text {rms }}\left(r_{1}\right) \equiv \sqrt{\left[i\left(r_{1}, t\right)^{2}\right)-\left(l_{\text {beam }}\left(r_{1}, t\right)^{2}\right\rangle}
$$

where the angular brackets indicate an ensemble average.

Consider now the DC-normalized correlation function for two radially separated points in the plasma, $r_{1}>r_{2}$ :

$$
R\left(r_{1}-r_{2}\right) \equiv \frac{\left\langle\left(\mid r_{1}, t\right) \tilde{I}\left(r_{2}, t\right)\right\rangle}{\operatorname{IDC}\left(r_{1}\right) I_{D C}\left(r_{2}\right)}=\frac{\left[I_{\text {plasma }}\left(r_{1}, t\right) \tilde{I}_{\text {plasma }}\left(r_{2}, t\right)\right)+\left[I_{\text {beam }}\left(r_{1}, t\right) \tilde{I}_{\text {beam }}\left(r_{2}, t\right)\right)}{\operatorname{IDC}\left(r_{1}\right) I_{D C}\left(r_{2}\right)}
$$

where the subscript 'DC' refers to the time averaged component of the signal. The cross terms in Eqn. 
6 are not correlated and thus go to zero. The locally induced fluctuations are also uncorrelated at large separations, i.e.,

$$
\left\langle\left(I_{\text {plasma }}\left(r_{1}, t\right) \tilde{I}_{\text {plasma }}\left(r_{2}, t\right)\right) \rightarrow 0 \text { as }\left|r_{1}-r_{2}\right| \rightarrow \infty\right.
$$

In practice, for Eqn. 7 is a good approximation when the radial separation is greater than 2-3 times the radial correlation length of the local fluctuations. This typically translates to greater than about $5-10 \mathrm{~cm}$ for typical TFTR discharges. Unlike the locally induced intensity fluctuations, the edge-induced beam density fluctuations are correlated at large radial separations. In fact, the DC-normalized amplitude of the edge-induced fluctuations is approximately independent of the radial separation (this is the reason the DC-normalized correlation function is used here rather than the RMS-normalized correlation coefficient) so that

$$
\frac{\tilde{I}_{\text {beam }}\left(r_{1}, t\right)}{\operatorname{lDC}\left(r_{1}\right)}=\frac{\tilde{I}_{\text {beam }}\left(r_{2}, t\right)}{\operatorname{lDC}\left(r_{2}\right)}
$$

Together, equations 5, 6, 7 and 8 give the desired result for the local, RMS fluctuation amplitude, corrected for edge-induced beam density fluctuations.

$$
i_{r m s}\left(r_{1}\right)=\sqrt{\left\langle\delta I\left(r_{1}, t\right)^{2}\right\rangle-I D C\left(r_{1}, t\right)^{2} R\left(r_{1}-r_{2} \mid\right)}
$$

It is this corrected $\tilde{I}_{\mathrm{rms}}$ which is related to the local plasma density fluctuations amplitude, $\tilde{n}_{\mathrm{rms}}$, via the atomic models. As an example from TFTR, Fig. 13 shows a radial profile of $\tilde{n}_{r m s} / n$ from a typical supershot both with and without this correction. It can be seen that deep in the plasma interior, where the local fluctuation amplitude is small, the uncorrected profile saturates at small $\mathrm{r} / \mathrm{a}$ because of edge induced fluctuations while the corrected profiles show that the local fluctuation amplitude actually continues to decrease..Note that the level of the edge-induced fluctuations in the core is about $0.2 \%$ which is quite good agreement with the simulation results.

A similar correction can be applied to the correlation function to derive a local plasma turbulence correlation function 4 :

$$
R_{\text {corr }}\left(r_{1}-r_{2}\right)=R\left(r_{1}-r_{2}\right)-R\left(r_{1}-r_{3} \mid\right)
$$

where $R_{\text {corr }}$ is the corrected correlation, $\left|r_{1}-r_{2}\right|$ is less than, and $\left|r_{1}-r_{3}\right|$ is greater than 2-3 times the 
local correlation length. Any residual r eutral beam npple which is not removed by the comb filtration also has very long radial correlation lengths and is thus also removed by these techniques. Note, however, that this technique presumes that points $r_{1}, r_{2}$, and $r_{3}$ are collinear with the neutral beam. More sophisticated techniques which correct for non-local beam density fluctuations when collinear data are not available are under development.

\section{Overview of Experimental Results}

In the 1990 and 1991 experimental campaigns, BES observations were made for a variety of TFTR plasma regimes. In particular, a systematic study was made of turbulence characteristics in a standard L-mode plasma, while less intensive observations were made of supershots, low $I_{P}$, high $\beta_{P}$ and $\mathrm{H}$ mode discharges. Initial emphasis was given to a detailed documentation of a standard L-mode plasma as a benchmark for BES turbulence observations. Following this effort, several run days were dedicated though 1991 to delineate the effects of rotation on the measurements and verify the localization of BES measurements. In the following sections we summarize some of the main results from these studies, including significant and unique measurements of the internal structure of the D-T relevant Toroidal Alfvén Eigenmode.

\section{Diagnostic Comparisons and Self Consistency}

One of the main goals for BES during the past two experimental campaigns has been a study of possible systematic effects and cross-comparisons with other fluctuation diagnostics in order to verify the performance of BES. Comparisons have been perforned with Langmuir probes on Phaedrus-T, and with microwave scattering, reflectometry and Mirnov coil arrays on TFTR.

Figures 14 and 15 show the autopower spectra and radial profile of $\tilde{n}_{r m s} / n$ from BES and Langmuir probes in Phaedrus-T. In both cases the agreement is quite good, which verifies that BES can accurately measure local density fluctuations. ${ }^{8}$

Comparisons with microwave scattering are more complicated since the two diagnostics have different $\mathbf{k}$-sensitivities and spatial resolutions. However, it is found that the phase velocities determined by the two diagnostics are in reasonable agreement. ${ }^{11}$

Figure 16 shows the results of a preliminary comparison between the microwave reflectometer and BES on TFTR. Note that the shape of the two curves is very similar though the reflectometer values are a factor of about 2.5 times higher than those $f_{1}, \mathrm{~m}$ BES. This is not totally unexpected since there is some question as to whether the simple 1-dimensional wave model used to interpret the reflectometer 
data actually yields the correct normalization (though the shape of the curve should be correct).

In general, agreement between BES and the Mirnov coil arrays on TFTR is quite good in the sense that the low $\mathrm{m} / \mathrm{n}$ MHD modes to which the Mirnov's are sensitive are also observed by BES (BES often observes higher $\mathrm{m} / \mathrm{n}$ coherent modes which are not clearly detected by the Mirnov coils). Figure 17 shows the autopower spectrum of the Toroidal Alfvén Eigenmode (TAE) from BES and the coherence between the BES and Mirnov signals. Near the TAE frequency the coherence is well above the noise level. Note that perfect coherence $(\gamma=1)$ is not expected here since the Mirnov coils' spatial response is global while the BES spatial response is local.

In addition to these diagnostic comparisons, tests of the self-consistency of the BES data have been performed. For example, if the plasma bulk toroidal rotation is varied by changing the neutral beam applied torque on the plasma (at constant total power) then the autopower spectrum should be Doppler shifted either up or down in frequency, depending on the direction of rotation. The Doppler-shifted autopower spectrum is given by $S\left(\omega^{\prime}\right)=S\left(\omega-\vec{k}_{\theta} \cdot \vec{v}_{\theta}\right)$ where $\omega$ is the angular frequency in a given frame of reference, $\omega$ ' is the frequency in a frame rotating at a relative toroidal velocity of $v_{\phi}$ and $v_{\theta}$ is the poloidal (i.e., vertical) component of the toroidal velocity, $v_{\theta}=v_{\phi} r / q R$. Note that only the signal due to real plasma fluctuations will show this Doppler shift. Systematic noise (e.g., electromagnetic pickup, amplifier noise, photon statisical fluctuations, intrinsic fluctuations in the neutral beam density, etc.) will not show a Doppler shift.

Figure 18 shows the autopower spectra for discharges with co, counter and nominally balanced injection. These spectra do show a clear Doppler shift with the co-spectrum being shifted up in frequency (relative to the balanced injection case) and the counter-spectrum being shifted down in frequency. This is a clear indication that the dominant part of the observed signal does indeed reflect local plasma fluctuations rather than some source of systematic noise.

\section{L-mode Fluctuation Characteristics}

Other experiments have begun to characterize long-wavelength turbulence in TFTR auxiliary heated plasmas, with an initial emphasis on standard L-mode discharges. The autopower spectrum in the Lmode is strongly concentrated at low frequencies (Fig. 11). Figure 19 shows the RMS fluctuation amplitude versus radius in an L-mode discharge. Note the large fluctuations near the edge $(r / a>0.9)$ and the rapid drop in fluctuation level in the core. In the interior a mixing length expression $\left(\tilde{n}_{\mathrm{rms}} / \mathrm{n} \approx \mathrm{L}_{\mathrm{d}} / \mathrm{L}_{\mathrm{n}}\right)$ predicts the right order of magnitude for the density fluctuations which implies that the fluctuations may be in the strong turbulence regime (as opposed to the weak turbulence or quasilinear regimes). This is consistent with the observation that $\Delta \omega / \omega \approx 1$ which also implies strong turbulence. 
The fluctuations near the edge are significantly larger than predicted by the mixing length model. This result has been seen in a number of experiments besides TFTR and may indicate that additional sources of instability are important near the edge (radiation or ionization drive, for instance).

The RMS core fluctuation level in the L-mode is found to scale with the injected neutral beam power (and thus scales inversely with the global energy confinement time, $\tau_{E}$ Fig. 20(a)). In contrast, the fluctuation level at the edge (Fig. 20(b)) is essentially independent of the injected power and the global confinement time. This indicates that L-mode energy confinement may be determined by turbulent transport in the core and is relatively independent of turbulence at the edge. However, there is some ambiguity since the RMS fluctuation levels are not necessarily monotonically related to the local energy flux (that is, changes in the phase and coherence between the density fluctuations and potential and temperature fluctuations can change the local heat flux without a change in $\tilde{n}_{\text {rms }} / \mathrm{n}$ ). To further quantify this observation it will be necessary to assume a particular model for the turbulent fluctuations in order to relate $\tilde{n}_{r m s} / n$ to the local heat flux. At present, these observations are circumstantial evidence that the observed core turbulence has a causal relationship to the global confinement in the Lmode.

Time-delay correlation analysis has been used to determine the poloidal group velocity of the fluctuations in the laboratory frame of reference. It is desirable to transform the measured velocity into the plasma frame of reference so that the velocity can be compared directly with theoretical predictions. The group velocity in the plasma-frame is given by $v_{g}^{p l}=v_{g}^{l a b}-v_{\theta}$ where $v_{\theta}$ is the poloidal velocity of the plasma. In the case where the actual poloidal rotation is damped, and the toroidal correlation length of the fluctuations is large there is an effective poloidal velocity due to the toroidal rotation velocity and the field line helicity: $v_{\theta}=v_{\phi} r / q R$. Figure 21 shows the plasma-frame group velocities derived under this assumption for a number of discharges with a range of toroidal velocities. The laboratory-frame group velocity is dominated by the toroial rotation. The plasma frame velocity is much smaller and is consistent with drift-wave propagation at the diamagnetic velocity. It appears that the plasma frame propagation tends to be in the ion-diamagnetic direction. However, there are several uncertainties in the transformation: the error bars on $v_{\phi}$ from Charge Exchange Recombination Spectroscopy (CHERS) are large; there is an additional uncertainty in the safety factor, $q$; and it has not been explicitly verified that the intrinsic poloidal rotation is damped in these discharges. Thus, within the error bars of the transformation the plasma-frame group velocity could be zero or even in the electron-drift direction. Work is in progress to reduce this uncertainty.

A similar analysis in the radial direction shows no evidence of radial propagation. The typical radial correlation length is on the order of $2 \mathrm{~cm}$. Figure 22 shows the radial correlation length as a function of radial position in an L-mode plasma. There is no strong scaling of the correlation length with radius. This is consistent with the observation that, in the L-mode, the density and temperature scale lengths 
are nearly constant in the core. The decorrelation times are on the order of 10-100 msec (depending on plasma conditions and radius)

In summary, initial characterizations of long-wavelength fluctuations during a standard L-mode show radially fixed, $\sim 2 \mathrm{~cm}$ structures which propagate slowly (if at all) in the poloidal direction. The fluctuations seem to be strongly turbulent and have decorrelation times on the order of 10-100 msec. The poloidal group velocity is on the order of, or less than, the diamagnetic velocity. The RMS fluctuation amplitude in the core is in rough agreement with mixing length estimates.

\section{General Characteristics of Long-Wavelength Density Fluctuations}

Observation of density fluctuations in regimes other than the standard L-mode (e.g., supershots and high $\beta_{\mathrm{P}}$ discharges) have found that a number of characteristics are generally similar to those seen in the standard L-mode. In balanced injection discharges, the autopower spectrum is always concentrated at low frequencies, $f<100 \mathrm{kHz}$ (Fig. 23). Edge plasma fluctuations (r/a $>0.9$ ) are typically on the order of $10-20 \%$. These strong edge fluctuations decrease sharply within a few centimeters of the limiter. Within the core there is a more gradual decrease in the fluctuation level with typical fluctuation levels in the range of $0.1-1 \%$. The observed spectra in rotating discharges always show strong Doppler shifts (which indicates that power is not concentrated very near $k_{\perp} \approx 0$ ). More detailed analysis and comparison of regimes other than the standard L-mode is in progress.

\section{Observations of Coherent and Quasi-Coherent MHD Modes}

While the primary experimental focus of BES has been diagnostic validation and characterization of turbulent density fluctuations, the system has also made some interesting observations of MHD phenomena, including the Toroidal Alfvén Eigenmode (TAE), tearing modes, quasi-coherent modes at high SP, $_{\mathrm{P}}$ and Edge Localized Modes (ELMs). BES, in conjunction with conventional Mirnov array analysis, provides a powerful tool for the study of MHD phenomena. BES can provide spatially localized measurements of the poloidal spectra and radial mode structure while the Mirnov arrays are used to identify the toroidal mode numbers and confirm the magnetic character of the modes.

The TAE is a global MHD instability which is driven by the fast ion pressure gradient. Calculation have shown that the TAE may be excited by fusion alpha particles at reactor conditions. This could lead to the loss of fast alpha confinement and a consequent inability to sustain a thermonuclear burn.

The TAE has been excited in TFTR by neutral beam injection into a low toroidal field, high density plasma. 12 The evidence that the excited modes are in fact TAE consists primarily of three observations: (1) the mode frequency scales with the Alfvén velocity; (2) the radial mode structure is global, rather 
than localized near a particular flux surface (Fig. 24); and (3) the dominant poloidal harmonics at a given radius obey the relationship $m+1 / 2 \approx n q(r)$ (Fig. 25 ). The latter two observations were made by BES and are crucial in confirming that the observed modes are TAE. Figure 24 shows that the saturated plasma displacement is on the order of $0.5-1 \mathrm{~cm}$. This amplitude is expected to be large enough to cause fast ion losses and such losses are observed (each periodic burst of TAE oscillations is accompanied by a loss of fast ions). 12 It is expected that TAE experiments will be a major experimental activity for BES during D-T operation. It is particularly important to understand how the instability threshold and the mode amplitude scale with temperature.

In addition to the TAE experiments there have been a number of serendipitous observations of MHD phenomena. Figure 26 shows the autopower spectrum of a strong $m / n=4 / 3$ tearing mode in a high $\beta_{\mathrm{P}}$ discharge $\left(\beta_{\mathrm{P}} / \beta_{\text {Troyon }} \approx 0.8\right)$. This mode is observed clearly both by $\mathrm{BES}$ and the Mirnov coil arrays. The apparent large spectral width $(\Delta \omega / \omega \approx 0.15)$ in the time integrated autopower spectrum is due to time variation of the mode frequency as shown by the set of time-resolved spectra. Figure 27 shows the radial structure of the mode. The cross-phase profile shows a 180 phase shift across the island, as expected. The profiles of $\tilde{n}_{\mathrm{rms}}(\mathrm{r}) / \mathrm{n}(\mathrm{r})$ give the island width directly as $2.5 \mathrm{~cm}$. These modes have a significant local effect on the plasma transport. Figure 28 shows the increase in $\chi_{e}$ near a large magnetic island with an attendant flattening of the temperature profile.

Other quasi-coherent modes have been observed in high $\beta$ p discharges which are not readily seen by the Mirnov coils. It is hypothesized that these modes represent high $\mathrm{m} / \mathrm{n}$ MHD activity or perhaps MHD turbulence (to which the Mirnov coils are not sensitive because of the strong decay of the high-m fields outside the plasma: $\widetilde{\mathrm{B}} \sim \mathrm{r}-\mathrm{m}$ ). Further observation and analysis of this interesting regime is proposed in support of the Columbia University high- $\beta$ p experiments on TFTR. 


\section{Status of Work on Alpha-CHERS (1991-1992)}

The alpha-CHERS diagnostic is an optical spectroscopy system being developed to measure the spatial and temporal distribution of the lower energy $\left(\mathrm{E}_{\alpha}<0.5 \mathrm{MeV}\right)$ non-thermal alpha particle population using charge exchange recombination spectroscopy. In particular, it is optimized to observe spectral line emission from the $\mathrm{n}=4-3$ transition of $\mathrm{He}^{+}$at $\lambda=468.6 \mathrm{~nm}$ produced by charge exchange recombination reactions between the alpha particles and a heating neutral beam. This system complements other diagnostics that are being designed to observe the higher birthing-energy alpha population, such as gyrotron scattering and pellet injection.

\section{A. Progress to Date}

The basic optical system for the alpha-CHERS system has been defined, and fabrication of various components is underway. A schematic of the optical system design is shown in Fig. 29. Optical fibers carrying light from the new alpha-CHERS ports on TFTR are mounted in a precision-drilled mount plate, which is mounted on a $x-y-z$ translation stage. A relay lens pair couple the light from the fibers to a special fiber-to-slit optical coupling array. The light is also converted form $\mathrm{f} / 2$ to $\mathrm{f} / 4$ at this stage for optimal coupling to the spectrometer. The fiber-to-slit assembly was designed via optical ray tracing to map the ten circular fibers to a curved input slit geometry, which in turn corrects for line curvature in the spectrometer. The output focal plane is reduced by a factor of 3 via a coherent fiber taper, and then lens coupled to a slow-scan CCD array. Most of the first set of coupling optics are in house or expected imminently. The special input fiber assembly is scheduled for delivery in mid-May.

Two options exist for obtaining at least 2 spatial locations in the plasma. The fiber bundles corresponding to each of two locations in the plasma are individually imaged onto separate entrance slit assemblies to separate the spectra around the desired $\mathrm{He}^{+} 469 \mathrm{~nm}$ line at the focal plane. A blocking filter in each channel eliminates overlap of the spectra of interest on the detector plane. The second option is to divide a single input slit vertically, with each spatial channel imaged to $1 / 2$ of the input slit.

Extensive tests of a slow-scan CCD camera for application to alpha-CHERS were made. It was found that the relative calibration of the response across the camera could be calibrated to at least one part in $10^{4}$, well within our requirements (cf. Fig. 30). Based on these results, we chose to employ a slow-scan CCD camera for the first generation alpha-CHERS detector. Princeton Instruments, Inc. won the competitive bid and has been contracted to deliver two slow-scan CCD cameras with special 
back-thinned CCD chips for high quantum efficiency. Delivery is expected in early July 1992, but testing and programming of the control software is being done before delivery of the caniera heads.

It was determined that a $0.5 \mathrm{~m}$ focal length spectrometer with a high-sensitivity $\mathrm{CCD}$ array detector was needed for three purposes: 1) monitoring of the background bremsstrahlung at high resolution for detection of small interfering line spectra; 2) detailed line shape analysis of the thermal $\mathrm{He}^{++}$ash line which will dominate the alpha particle signal at line center; and 3) detection of broadband optical fiber fluorescence excited to high neutron fluxes during D-T operation on TFTR. The purchase of this spectrometer is presently out on bid, and delivery is expected before the end of June. The detector will be identical to the alpha-CHERS system to reduce programming and installation time.

Interfacing to the PPPL mechanical support group and computer division has started. In particular, work with the data acquisition and analysis engineers in the PPPL computer division has led to simplification of various interface and control functions. All physical manipulations of the hardware (e.g., wavelength changes, fiber bundle selection, etc.) will be implemented through programmable motor-driven micrometers, identical to those employed on the TFTR BES fiber switching assembly.

Assuming all component deliveries stay on schedule, we plan to install the first alpha-CHERS channels on TFTR in late July or early August of 1992. This is about one or two months later than originally scheduled, with most of the delay caused by the loss of the lead scientist on this project in January of this year. 


\section{References}

1. A.J. Wootton, B.A. Carreras, H. Matsumoto, K. McGuire, W.A. Peebles, Ch. P. Ritz, P.W. Terry, S.J. Zweben, Phys. Fluids B 2, 2879 (1990).

2. R.J. Fonck, P.A. Dupperrex, S.F. Paul, Rev. Sci. Instrum. 61, (1990).

3. R.J. Fonck, R.A. Ashley, R.D. Durst, S.F. Paul and G. Renda, 'Low-Noise Photodiode Detector for Optical Fluctuation Diagnostics', accepted for publication in Rev. Sci. Instrum.

4. R.D. Durst, R.J. Fonck, S.F. Paul, G. Cosby, H. Evensen, 'Density Fluctuation Measurements via Beam Emission Spectroscopy', submitted to Rev. Sci. Instrum.

5. R.J. Fonck, G. Cosby, R.D. Durst, T. Gibney, M. Thompson, S.F. Paul, 'Remote Operation of the TFTR BES Experiment from an Off-Site Location.', accepted for publication in Rev. Sci. Instrunm.

6. S. F. Paul and R.J. Fonck, Rev. Sci. Instrum. 61, 3496 (1990).

7. S.F. Paul, D. Cylinder, G. Drozd, R.J. Fonck, R.D. Durst, 'Mechanized Selection of Fiber Optic Arrays for Spectroscopic Measurements', accepted for publication in Rev. Sci. Instrum.

8. H.T. Evensen, D.A. Brouchous, D. Diebold, M. Doczy, R.J. Fonck and D.R. Nolan, 'Optical Fluctuation Measurements of Turbulence Using a Diagnostic Neutral Beam on Phaedrus-T", accepted for publication in Rev. Sci. Instrum.

9. T.A. Gianakon, J.D. Callen, R.J. Fonck, J.S. Kim, R.D. Durst, 'Effects of Edge Plasma Turbulence on Radial Correlation Length Measurements with BES', submitted to Rev. Sci. Instrum.

10. J.S. Bendat and A.G. Piersol, Random Data, J. Wiley (1986).

11. N. Bretz, R. Nazikian, S. Paul, R. Fonck, R.D. Durst, 'A Comparison of Fluctuation Spectra from Microwave Scattering and BES on TFTR', submitted to Rev. Sci. Instrum.

12. K.L. Wong, R.J. Fonck, S.F. Paul, D.R. Roberts, E.D. Fredrickson, R. Nazikian, H.K. Park, M. Bell, N.L. Bretz, R. Budny, S. Cohen, G.W. Hammet, F.C. Jobes, D.M. Meade, S.S. Medly, D. Mueller, Y. Nagayama, D.K. Owens and E.J. Synakowski, Phys. Rev. Lett. 66, 1874 (1991).

13. M. von Hellermann, private communication, 1991. 


\section{Appendix A: Papers from Previous Grant Period}

1) Internal Microturbulence Studies on DIII-D, TEXT and TFTR, W.A. Peebles, et al., IAEA Conference Proceedings, IAEA-CN-53/A-7-12, Washington DC (1990).

2) Plasma Fluctuation Measurements in Tokamaks Using Beam-Plasma Interactions, R.J. Fonck, P.A. Dupperrex, and S.F. Paul, Rev. Sci. Instrum., 61, 3487 (1990).

3) Beam Emission Spectroscopy Diagnostic for the Study of Turbulence in the Phaedrus-T Tokamak, Y.J. Kim, R.A. Breun, D.A. Brouchous, and R.J. Fonck, Rev. Sci. Instrum, 61, 3046 (1990).

4) Neutral Beam Emission Spectroscopy Diagnostic for Measurement of Density Fluctuations on the TFTR Tokamak, S.F. Paul, R.J. Fonck, Rev. Sci. Instrum., 61, 3496 (1990).

5) Long Wavelength Density Turbulence Measurements in TFTR Beam-Heated Discharges, R.J. Fonck, S.F. Paul, D.R. Roberts, Y.J. Kim, N. Bretz, et al., Proceedings of the 18th European Conference on Controlled Fusion and Plasma Physics, Berlin, Vol. I, 269 (June 1991).

6) Visible Charge Exchange Recombination Spectroscopy on TFTR, B. C. Stratton, R. J. Fonck, K. P. Jaehnig, N. Schechtman, and E. J. Synakowski, Proceedings of IAEA Technical Committee Meeting on Time-Resolved Two- and Three-Dimensional Plasma Diagnostics, Nagoya, Japan (Nov. 1990).

7) Excitation of Toroidal Eigenmodes in TFTR, K.L. Wong, R.J. Fonck, S.F. Paul, D.R. Roberts, et al., Phys. Rev. Lett. 66, 1874 (1991).

8 Investigation of Global Alfven Eigenmode Instabilities in TFTR, K.L. Wong, R.D. Durst, R.J. Fonck, and S.F. Paul, accepted for publication in Phys. Fluids B.

9) Measurements of the Radial Structure and Poloidal Spectra of the Toroidal Alfvén Eigenmode in TFTR, R.D. Durst, K.L. Wong, C.Z. Cheng, R.J. Fonck, E.D. Fredrickson, S.F. Paul, submitted to Phys. Fluids B.

10) Atomic Processes and Spectroscopic Techniques Applied to Fusion Plasma Diagnostics, R. J. Fonck, Atomic Processes in Plasmas, Ed. Y. Kim and R. C. Elton, AIP Conf. Proceedings 206, 122 (1990)

11) Measurements of Long-Wavelength Density Fluctuations in TFTR, S.F. Paul, N. Bretz, R. Durst, R.J. Fonck, E. Mazzucato, R. Nazikian, submitted to Phys. Fluids B.

12) Optical Fluctuation Measurements of Turbulence Using a Diagnostic Neutral Beam on Phaedrus-T, H.T. Evensen, D.A. Brouchous, D. Die'told, M. Doczy, R.J. Fonck and D.R. Nolan, accepted for publication in Rev. Sci. Instrum.

13) Low-Noise Photodiode Detector for Optical Fluctuation Diagnostics, R.J. Fonck, 
R.A. Ashley, R.D. Durst, S.F. Paul and G. Renda, accepted for publication in Rev. Sci. Instrum.

14) Mechanized Selection of Fiber Optic Arrays for Spectroscopic Measurements, S.F. Paul, D. Cylinder, G. Drozd, R.J. Fonck, R.D. Durst, accepted for publication in Rev. Sci. Instrum.

15) Effects of Edge Plasma Turbulence on Radial Correlation Length Measurements with BES, T.A. Gianakon, J.D. Callen, R.J. Fonck, J.S. Kim, R.D. Durst, accepted for publication in Rev. Sci. Instrum.

16) Density Fluctuation Measurements via Beam Emission Spectroscopy, R.D. Durst, R.J. Fonck, S.F. Paul, G. Cosby, H. Evensen, submitted to Rev. Sci. Instrum.

17) A Comparison of Fluctuation Spectra from Microwave Scattering and BES on TFTR, N. Bretz, R. Nazikian, S. Paul, R. Fonck, R.D. Durst, submitted to Rev. Sci. Instrum.

18) Remote Operation of the TFTR BES Experiment from an Off-Site Location, R.J. Fonck, G. Cosby, R.D. Durst, T. Gibney, M. Thompson, S.F. Paul, accepted for publication in Rev. Sci. Instrum.

19) Alpha-CHERS: A Spectroscopic Experiment to Detect Non-Thermal Alpha Particles on TFTR, B.C. Stratton, R.J. Fonck, Y.J. Kim, G. McKee, J. Schivell, T. Thorson, accepted for publication in Rev. Sci. Instrum.

20) Line Shapes in Charge Exchange Recombination Spectroscopy, Spectral Line Shapes Volume 6, Ed. Y. Kim and R. C. Elton, AIP Conf. Proceedings 216, 31 (1990)

21) Tests of High-Stability, Low-Noise Detectors for the TFTR alpha-CHERS Experiment, T. Thorson, R. Ashley, R.J. Fonck, and B.C. Stratton, accepted for publication in Rev. Sci. Instrum.

22) Measurement of Iron Transport in the TFTR Tokamak by Charge Exchange Recombination Spectroscopy, B.C. Stratton, E.J. Synakowski, P.C. Efthimion, R.J. Fonck, et al., Nucl. Fusion, 31, 171 (1991).

23) Measurements of Radial Profiles of $\mathrm{He}^{2++}$ Transport Coefficients on the TFTR Tokamak, E.J. Synakowski, B.C. Stratton, P.C. Efthimion, R.J. Fonck, et al., Phys. Rev. Lett., 65, 2255 (1990).

24) Intense Diagnostic Neutral Beam Development for ITER, D.J. Rej, I. Henins, R.J. Fonck, Y.J. Kim, accepted for publication in Rev. Sci. Instrum.

25) A Simple Multi-Foil Spectrometer for X-Ray Line Measurements, S. Hokin, R.J. Fonck, P. Martin, accepted for publication in Rev. Sci. Instrum.

26) PBX-M Research Progress: Approach to Second Stability, N. Sauthoff, et al., Conference Proceedings 13th IAEA Conf. on Plasma Phys. and Cont. Nucl. Fusion, IAEA-CN53/E-II-4, I, 709 (1990).

27) Image Reconstruction Methods for the PBX-M Pinhole Camera, A. Holland, E. T. Powell, and R.J. Fonck, Applied Optics, 30, 3740 (1991). 
28) Technique for Relating Internal Plasma Shape to Plasma Current Profiles in Noncircular Tokamaks, E. T. Powell, R. Kaita, and R.J. Fonck, Rev. Sci. Instrum., 61, 3301 (1990).

29) Control of Plasma Shape and Performance of the PBX-M Tokamak Experiment in High- $\beta_{t} /$ High- $\beta_{p}$ Regimes, R. E. Bell, et al., Phys. Fluids $B, 2,1271$ (1990).

29) Control of Plasma Shape and Performance of the PBX-M Tokamak Experiment in High- $\beta_{t} /$ High- $\beta_{p}$ Regimes, R. E. Bell, et al., Phys. Fluids $B, 2,1271$ (1990).

30) Iron Concentration Measurements on TFTR Using Charge Exchange Excited Lines of Helium-like Iron in the 200-700 $\AA$ Region, B. C. Stratton, R. J. Fonck, D. W. Johnson, H. Park, A. T. Ramsey, E. J. Synakowski, and J. Timberlake, Rev. Sci. Instrum., 61, 3113 (1990).

31) Charge Exchange Recombination Spectroscopy Measurements in the Extreme Ultraviolet Region of Central Carbon Concentrations during High Power Neutral Beam Heating in TFTR, B. C. Stratton, R. J. Fonck, A. T. Ramsey, E. J. Synakowski, B. Grek, K. W. Hill, D. W. Johnson, D. K. Mansfield, H. Park, G. Taylor, and P. M. Valanju, Nucl. Fusion, 30, 675 (1990). 


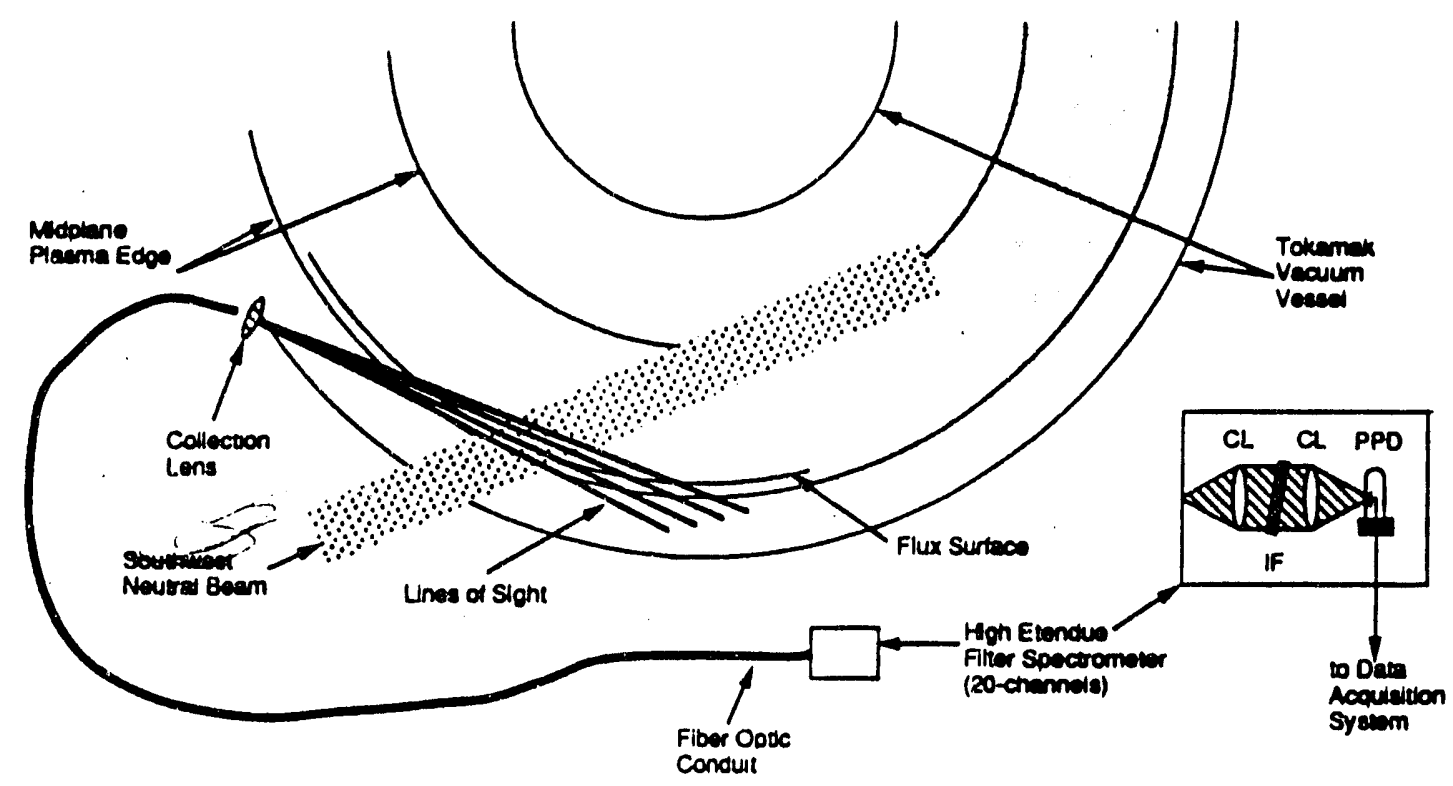

Figure 1. Schematic diagram showing the principle of Beam Emission Spectroscopy 


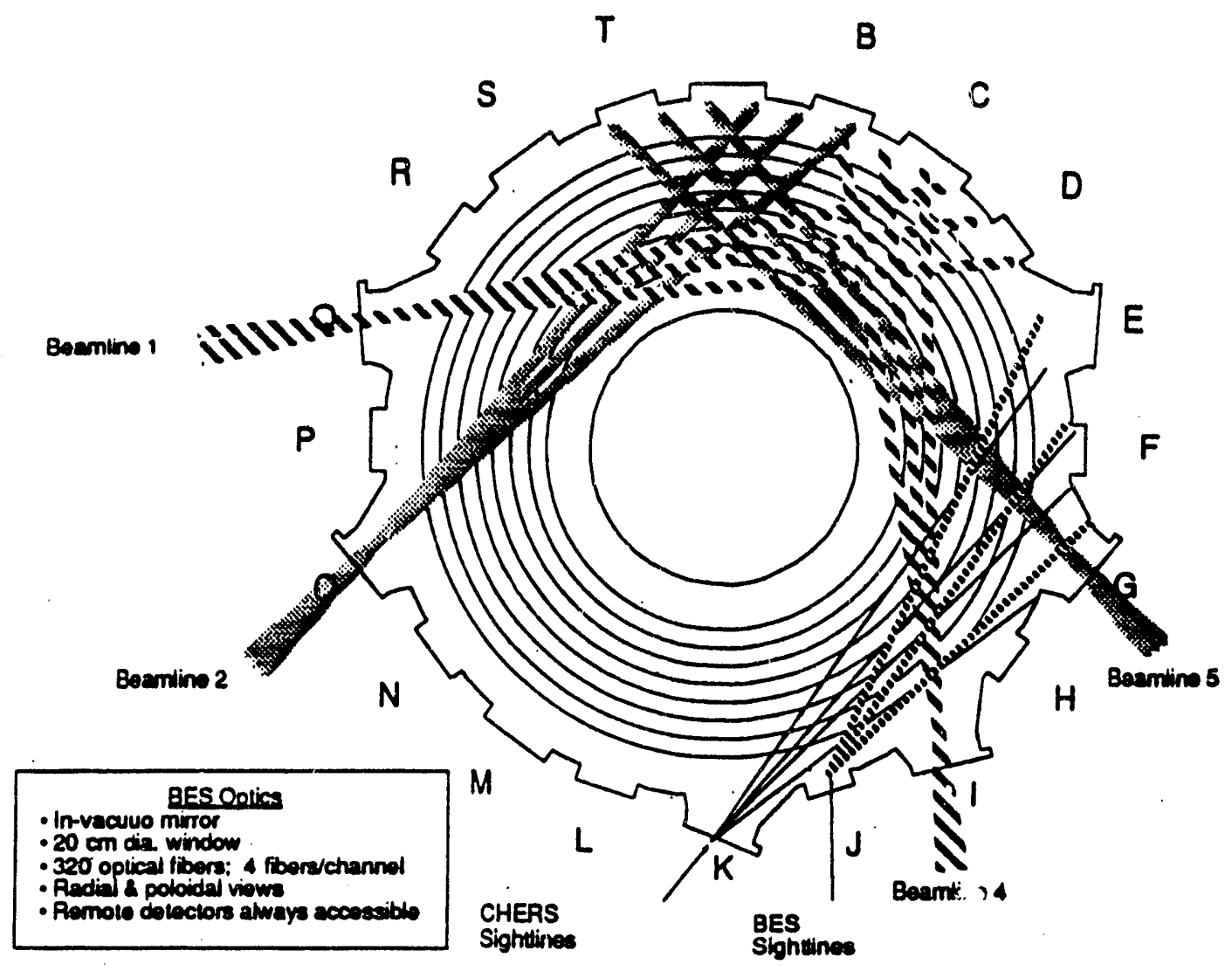

Figure 2. Plan view of TFTR neutral beam lines and BES sightlines.

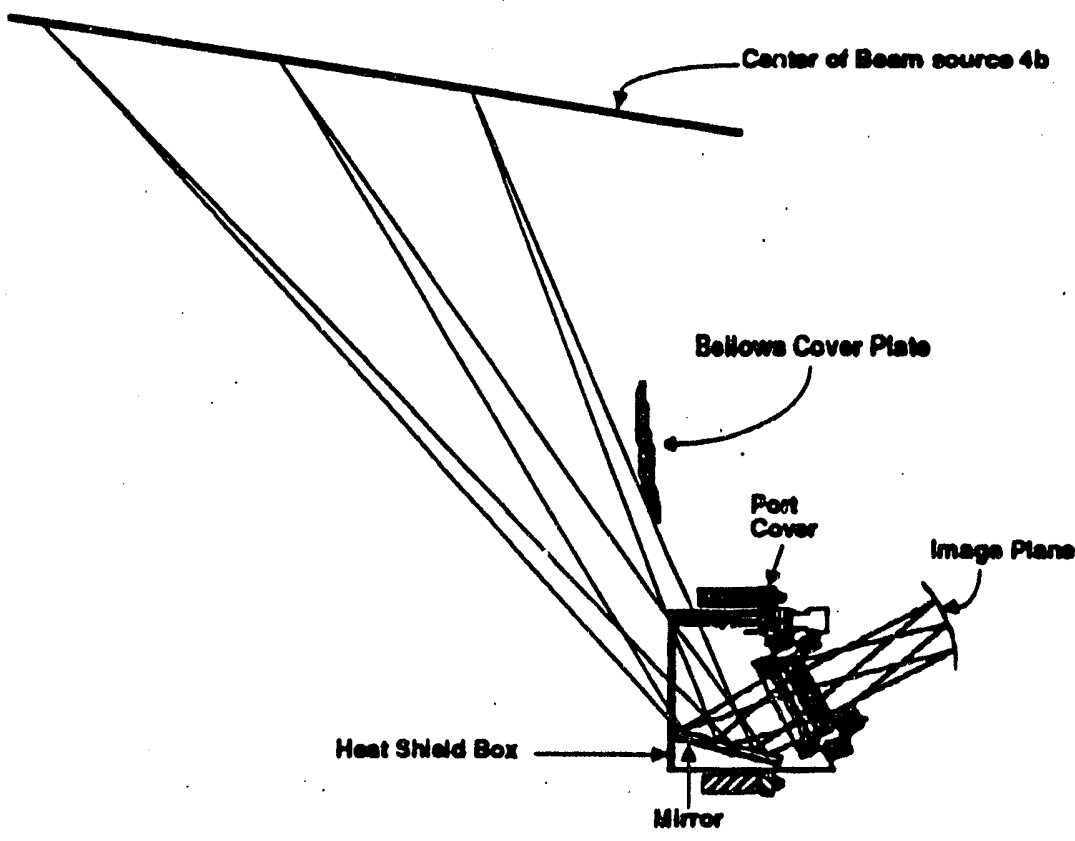

Figure 3. The BES collection optics on TFTR. 


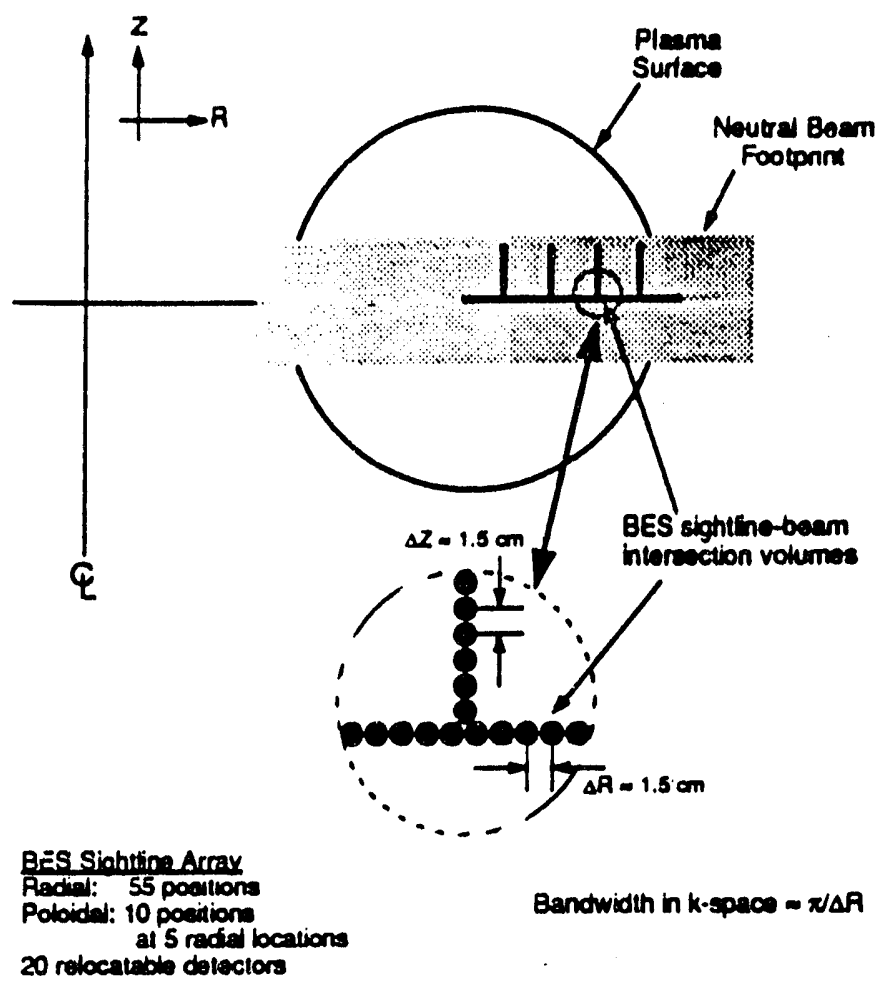

Figure 4. The array of poloidal and radial sightlines available to BES.

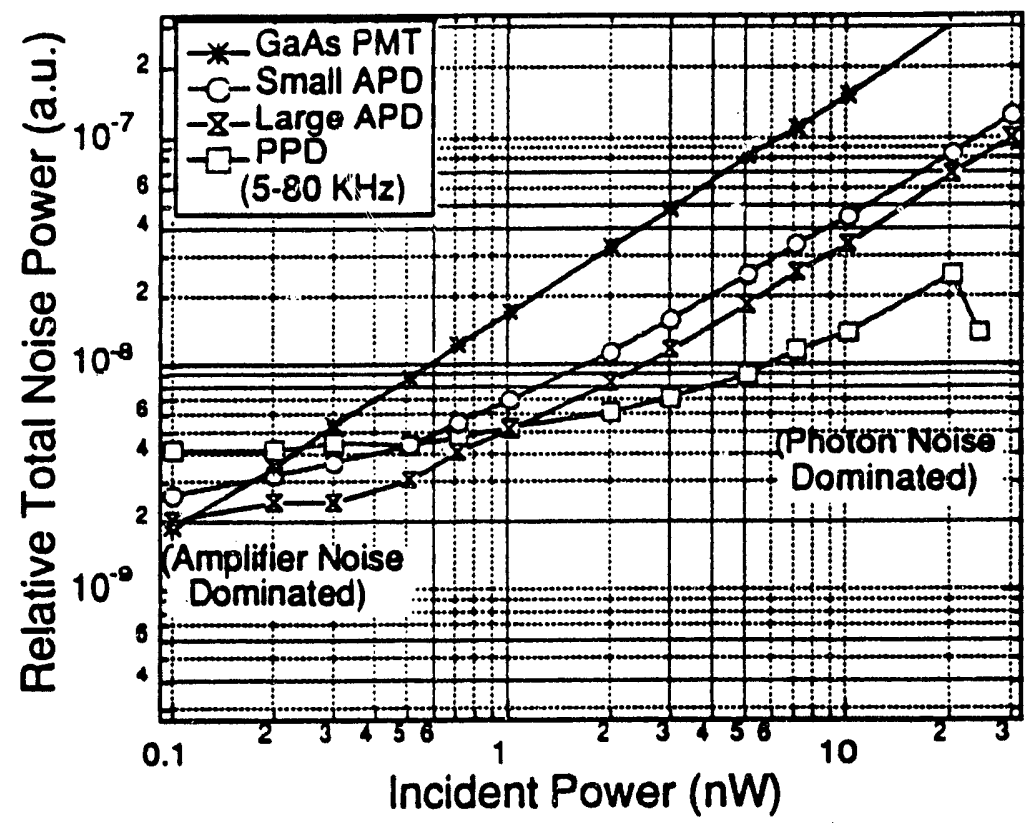

Figure 5. Total noise (amplifier plus photon) for several detectors (PMT, APD, PPD). The PPD is usefuli] for photon-noise-limited detection in the range of 1 to $20 \mathrm{nW}$ of input power. The PMT is about 15 times, and the APD about 4 times as noisy as the $P P D$ in this range. 

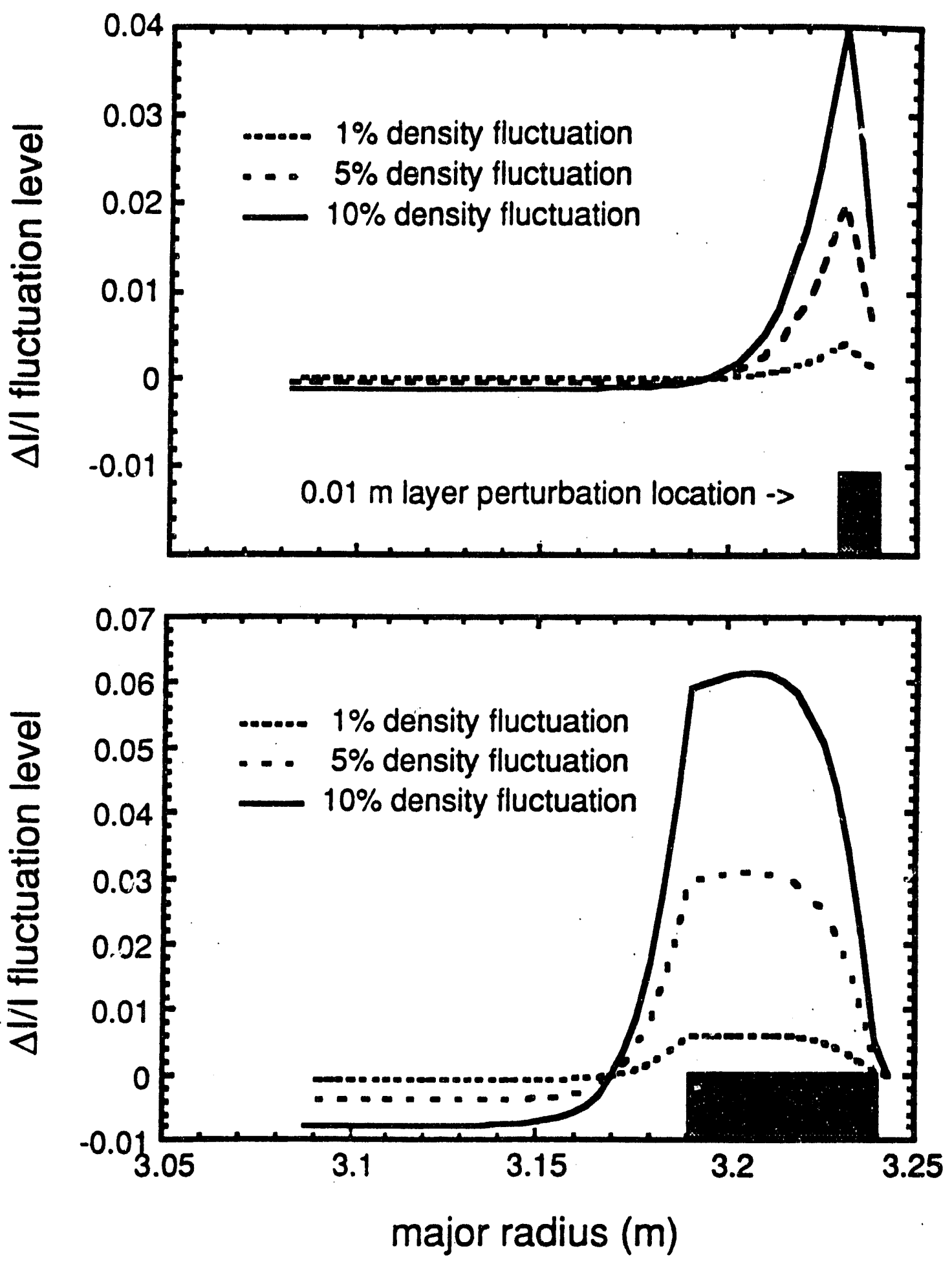

Figure 6. Simulated fluorescence fluctuations due to plasma density fluctuations of the indicated size and width (fef 7). 


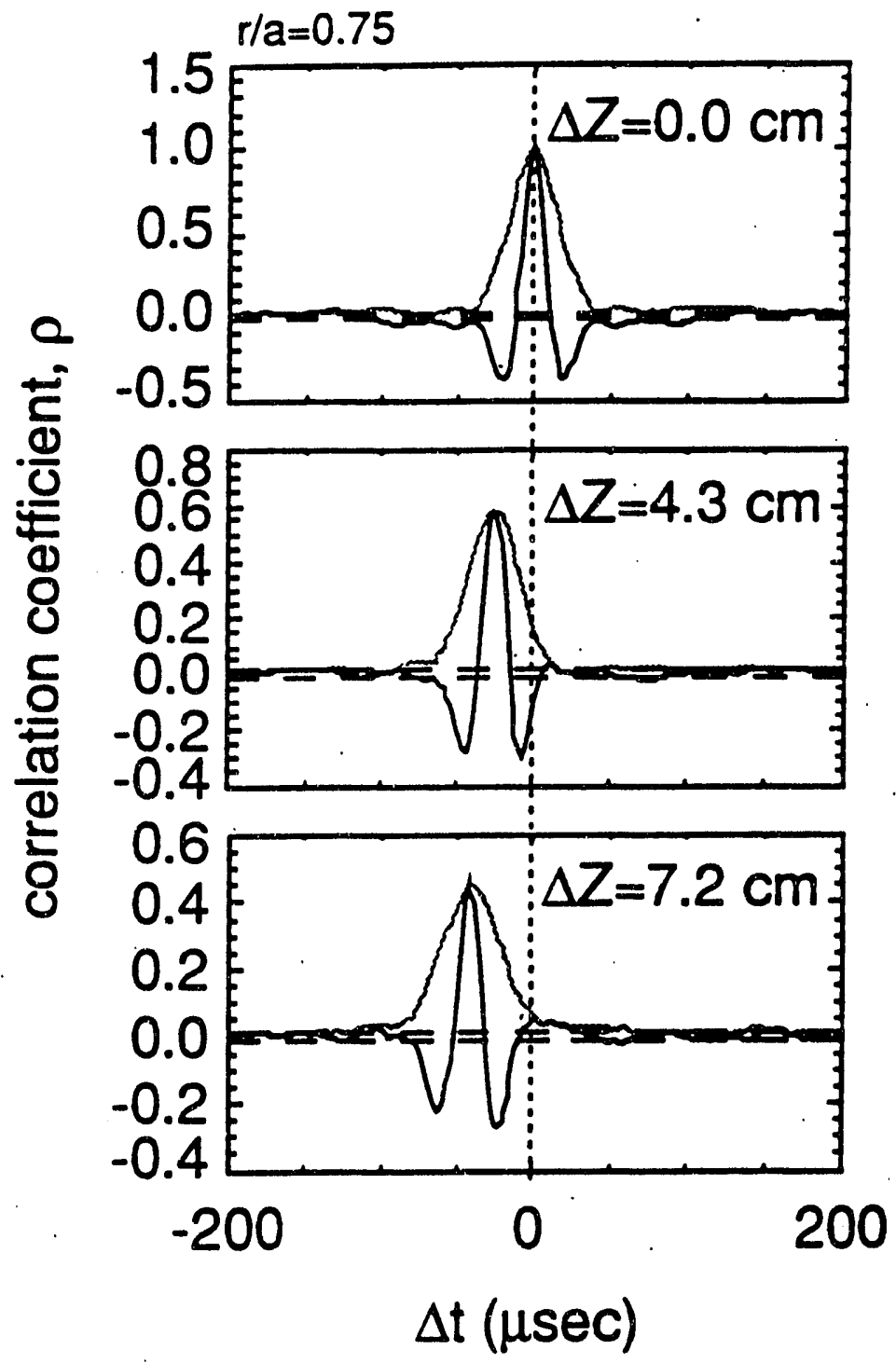

Figure 7. Typical poloidal cor: slation coefficient functions from a TFTR supershot (61292). 


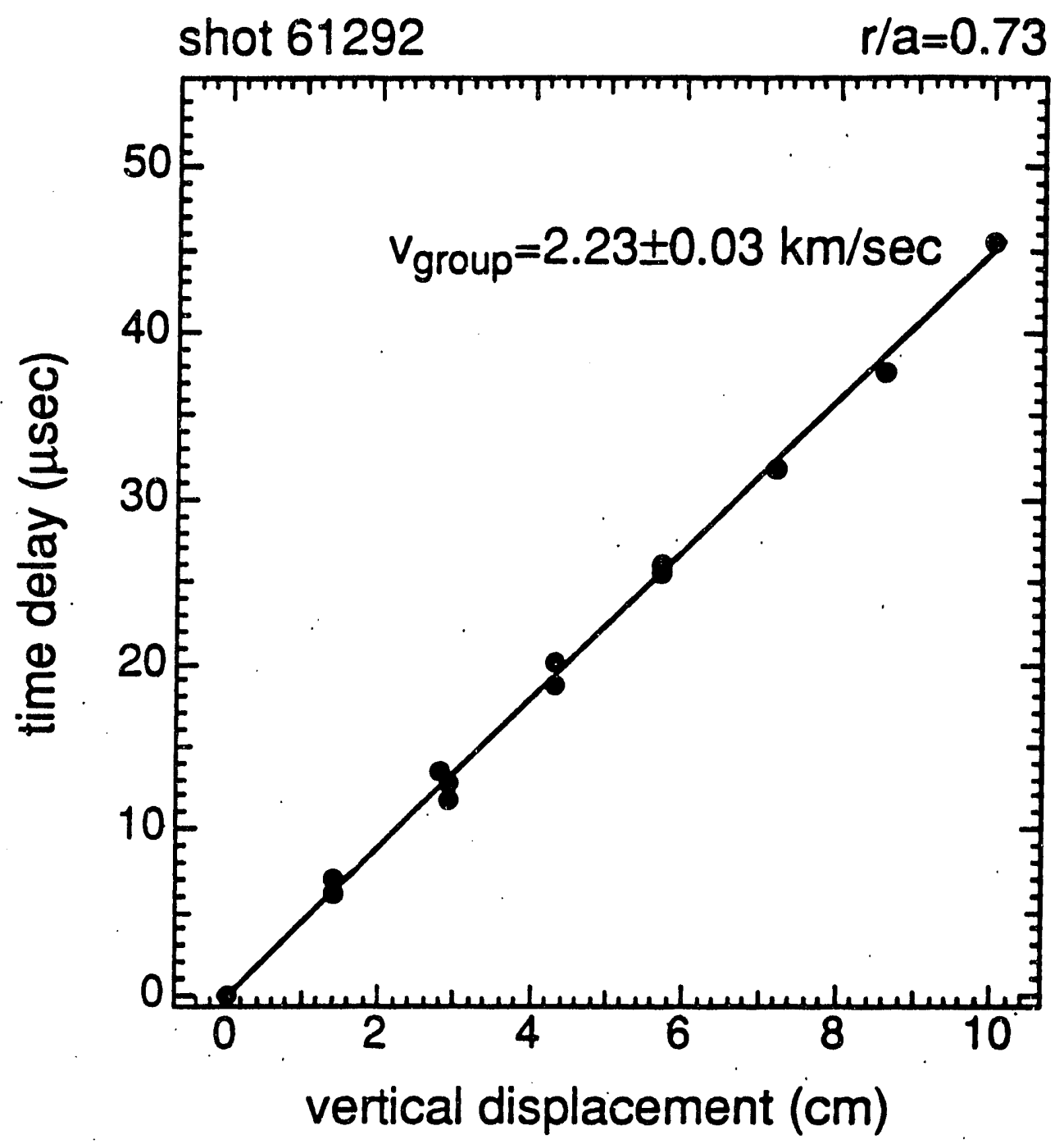

Figure - 8. The time delay of the peak of the coffelation coefficient function for each channel (Fig. 7) gives the group velocity of the mode. 


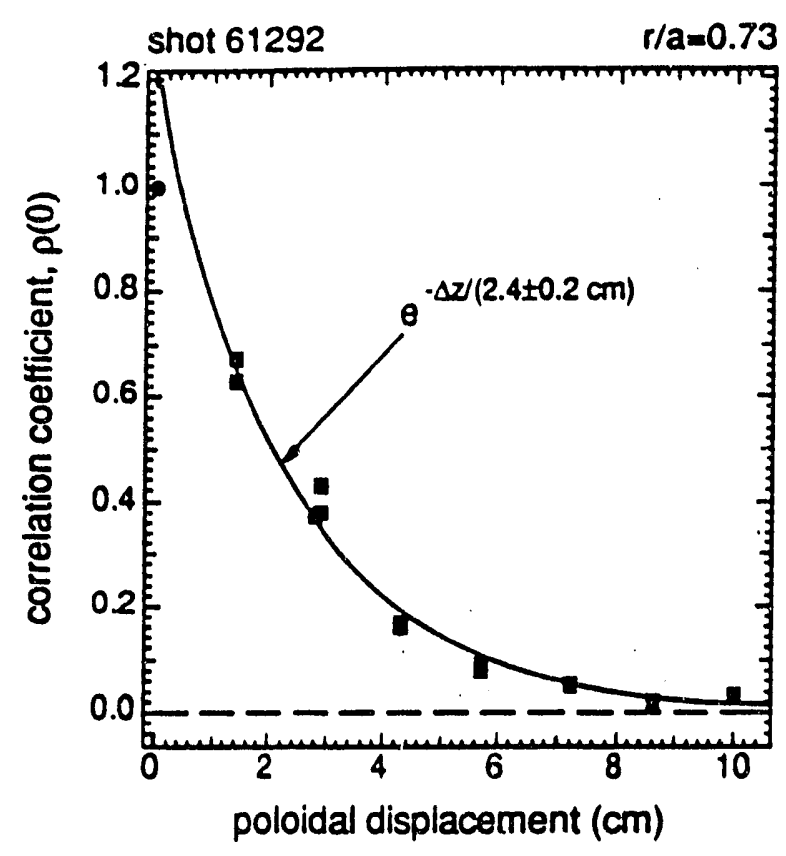

Figure 9. A typical profile of the correlation coefficient at zero time delay, $\rho(\Delta z, \Delta t=0)$. The correlation length is the e-folding distance of this profile $(2.4 \mathrm{~cm})$.

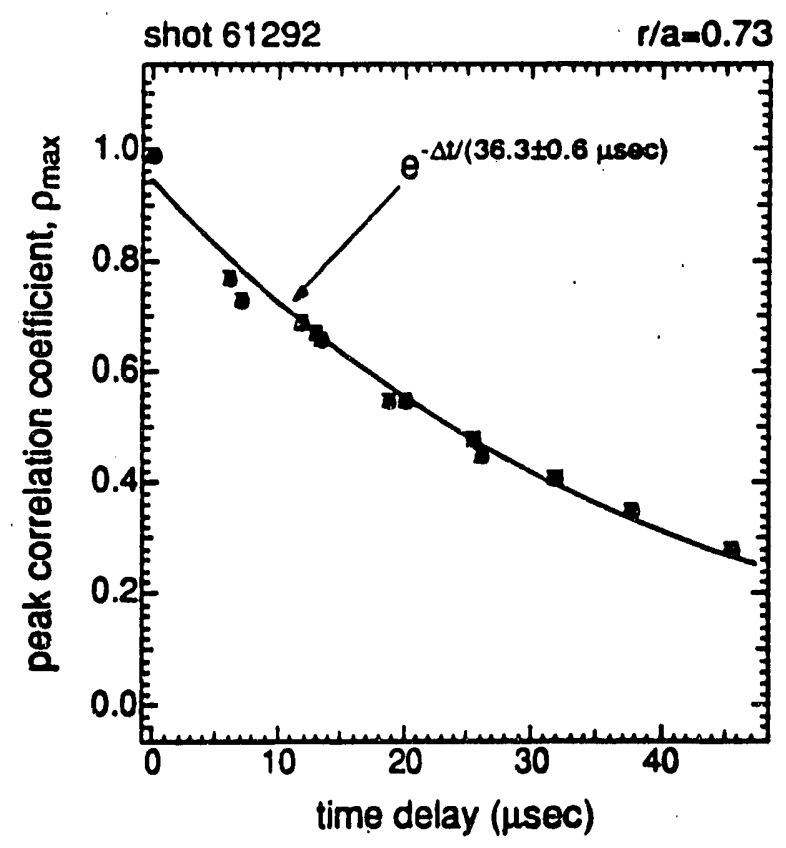

Figure 10. The decay of the peak value of the correlation coefficient function gives the decorrelation time which is a time scale of the plasma turbulence. 

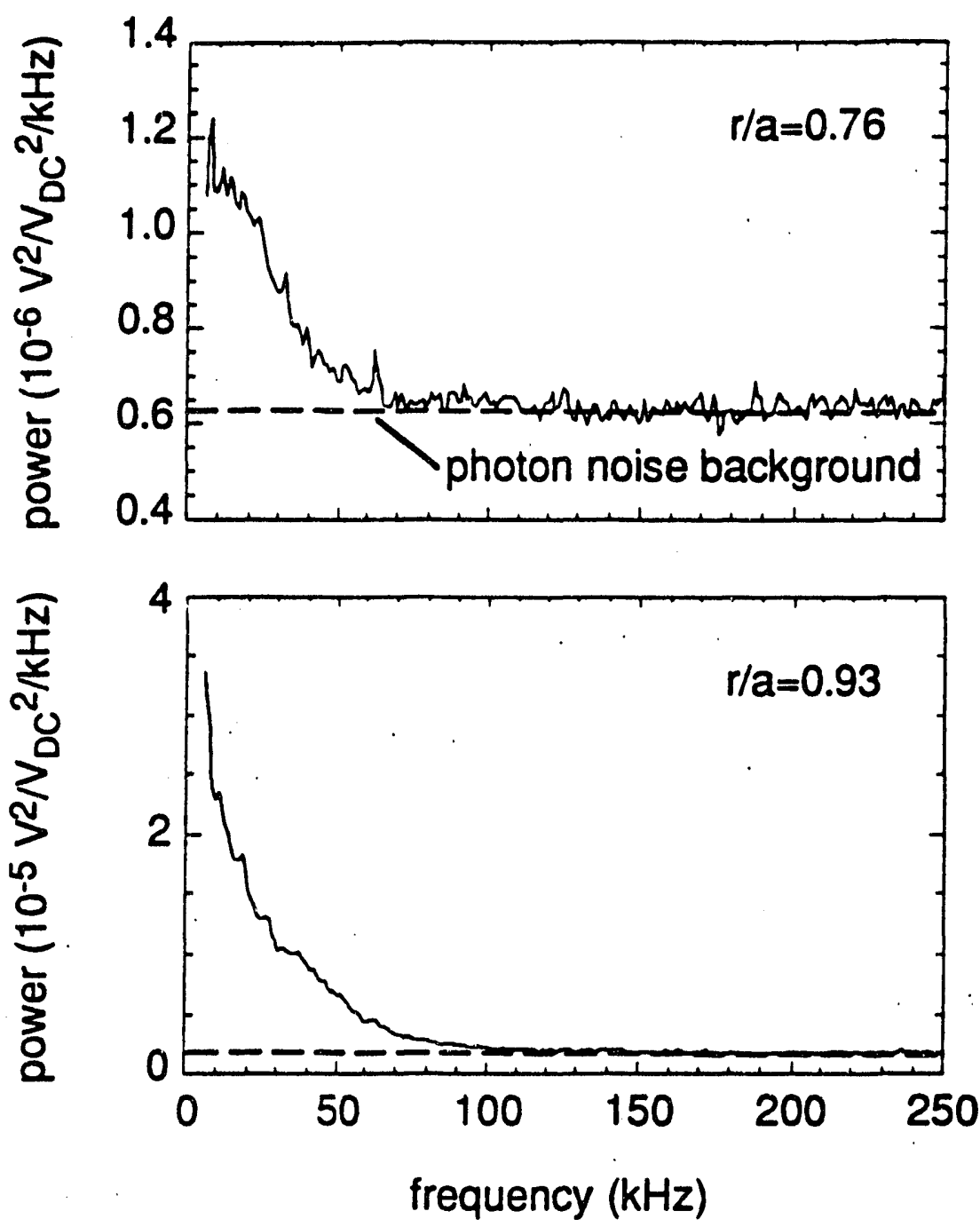

Figure 11. Typical L-mode power spectra. Balanced injection, $P_{\mathrm{BEAM}}=10 \mathrm{MW}$. Shot 56369. 


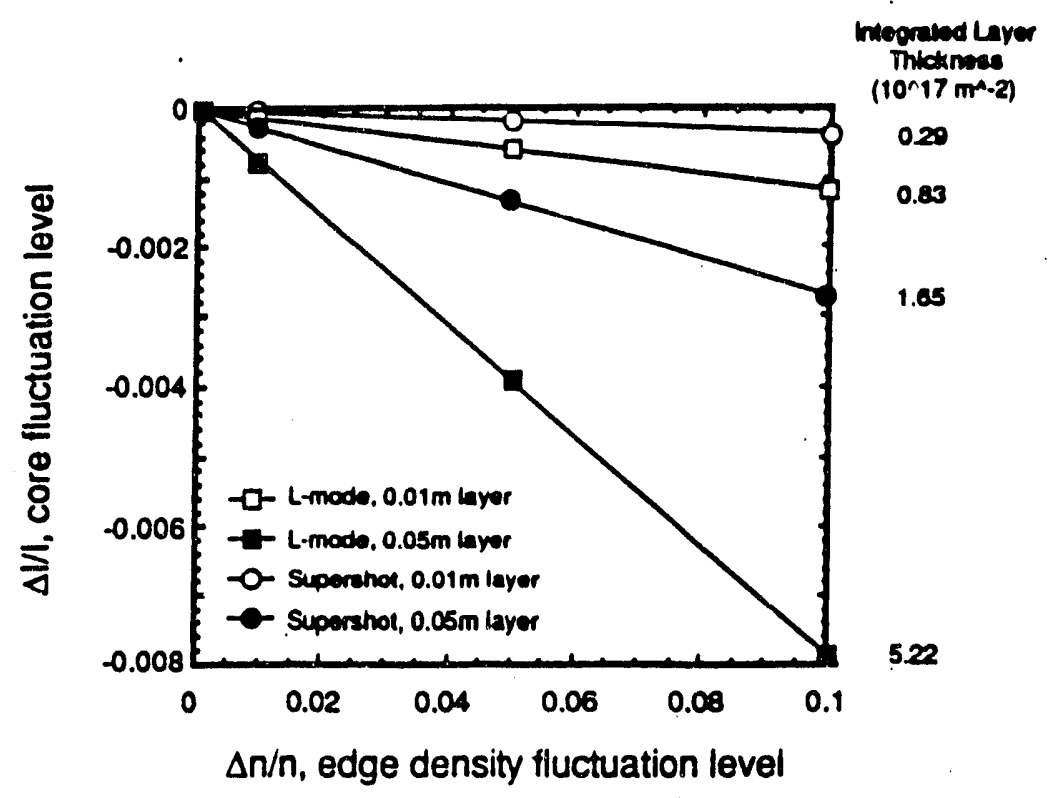

Figure 12. The edge-induced fluctuations in the core neutral beam fluorescence as a function of the edge fluctuation level for various assumed fluctuation sizes, plasma profiles (supershot or L-mode). Reference 7.

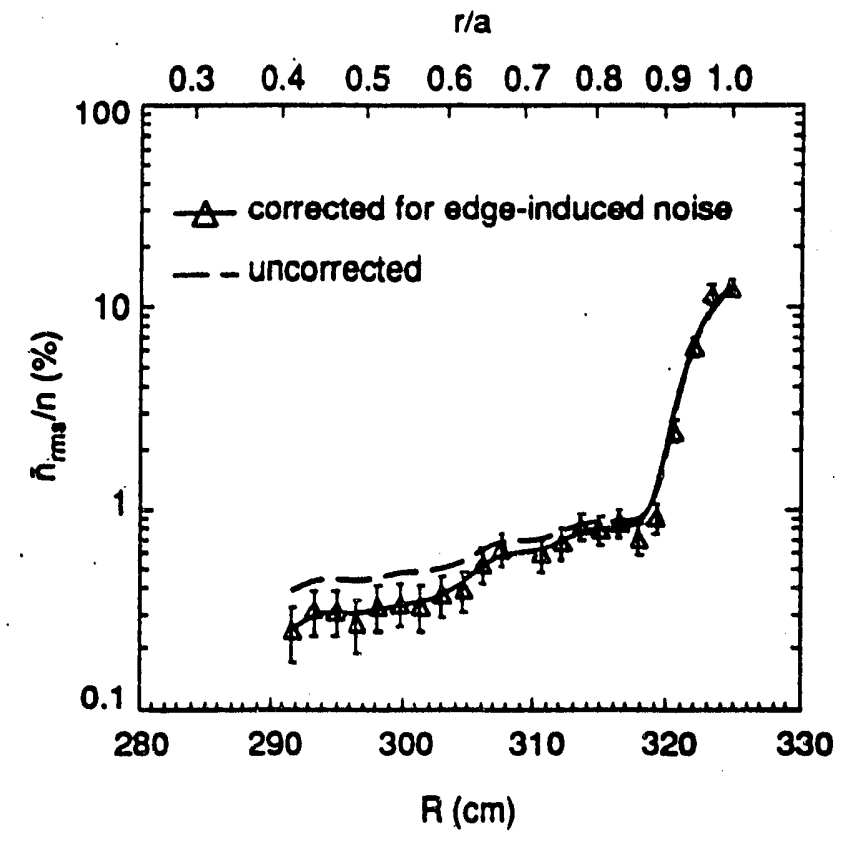

Figure 13. A profile of $\tilde{n}_{\mathrm{rms}} / \mathrm{n}$ for a typical supershot. The triangles have been corrected for edge-induced beam noise while the dashed line shows the uncorrected data. 

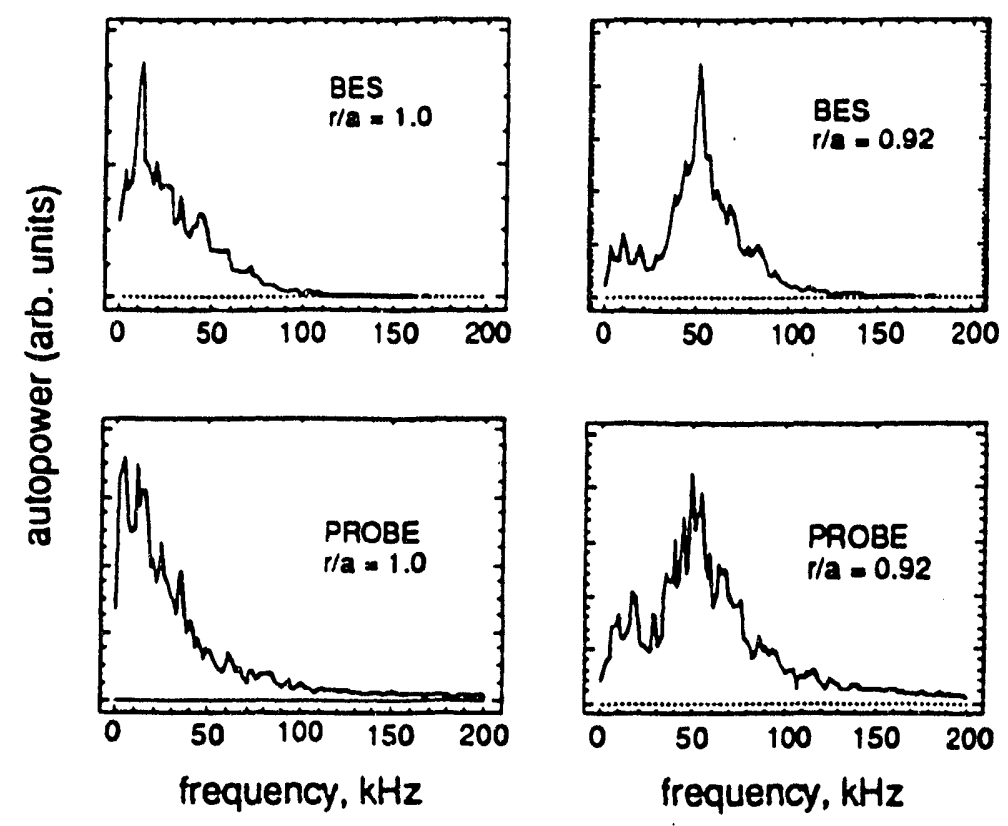

Figure 14. Autopower spectra from BES and Langmuir probes on Phaedrus-T.

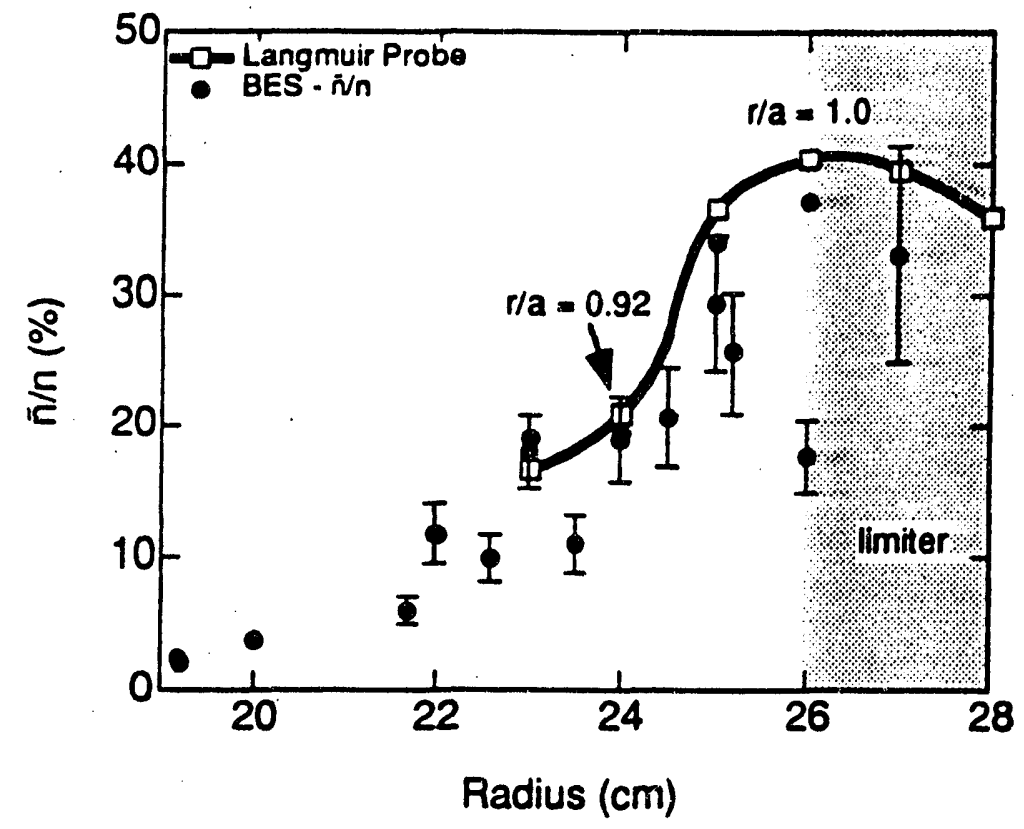

Figure 15. Acomparison of the RMS fluctuation amplitudes from BES and Langmuir probes near the edge of Phaedrus-T. 


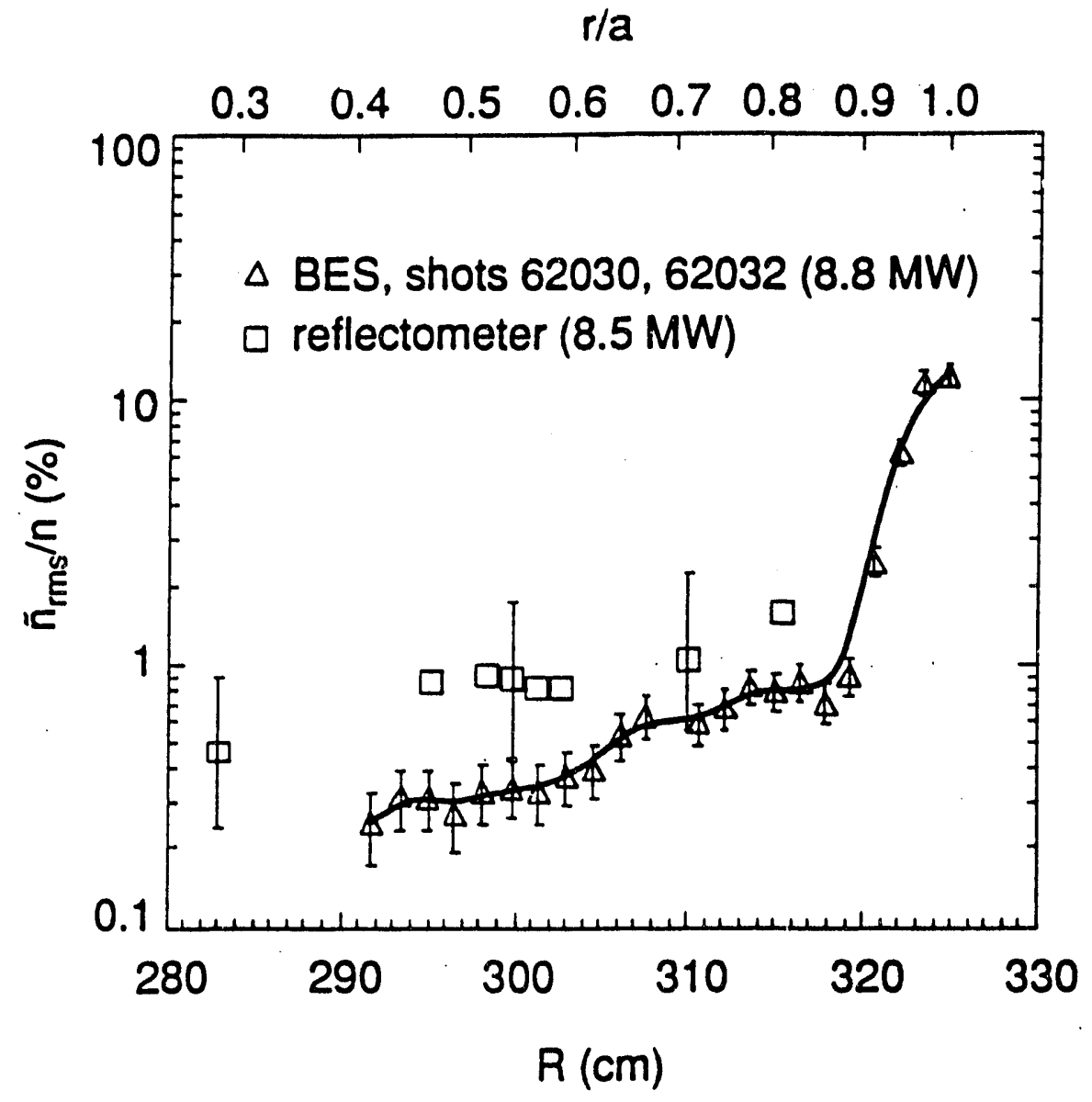

Figure 16. A comparison of $\tilde{n}_{r m s} / \mathrm{n}$ from BES and microwave reflectometry. Reflectometer data courtesy of E. Mazzucato, PPPL. 

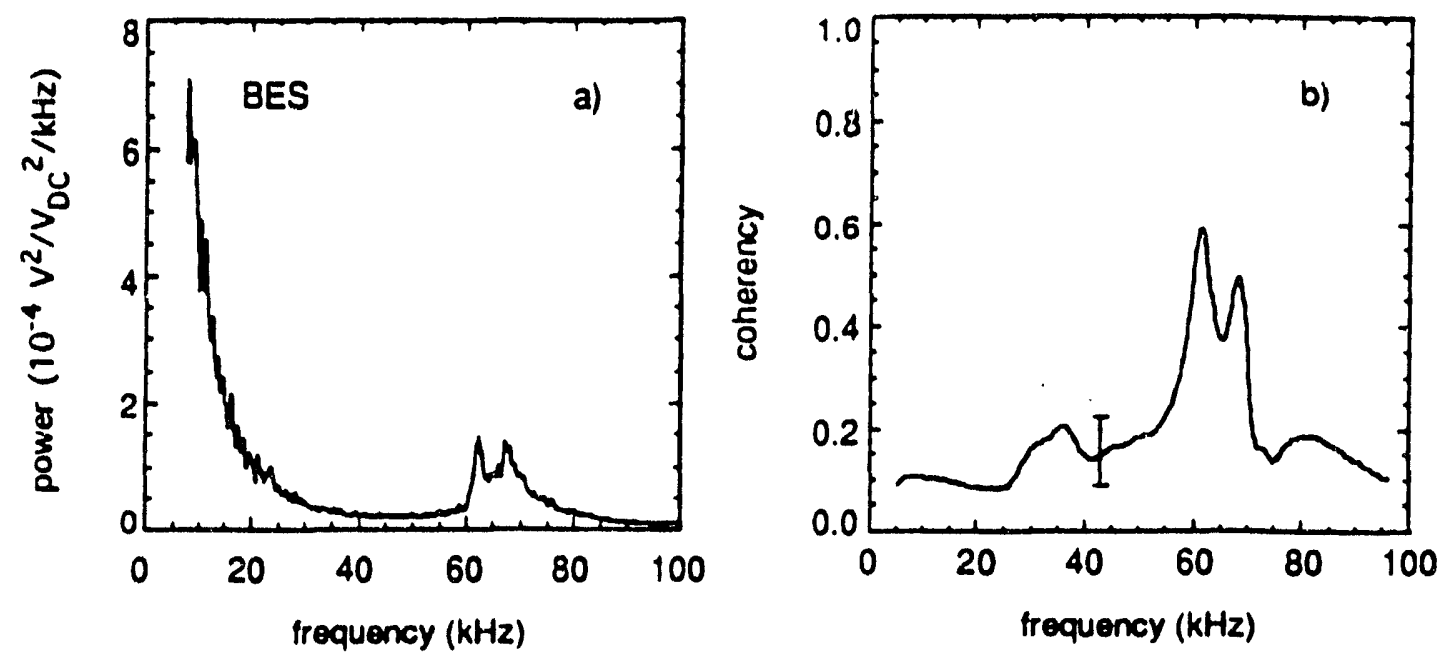

Figure 17. Autopower spectoum for a TAE discharge (a) and the coherency between BES and one of the Mimov coils (b). The coherency between the two signals is significant near the TAE frequency $(65 \mathrm{kHz})$.

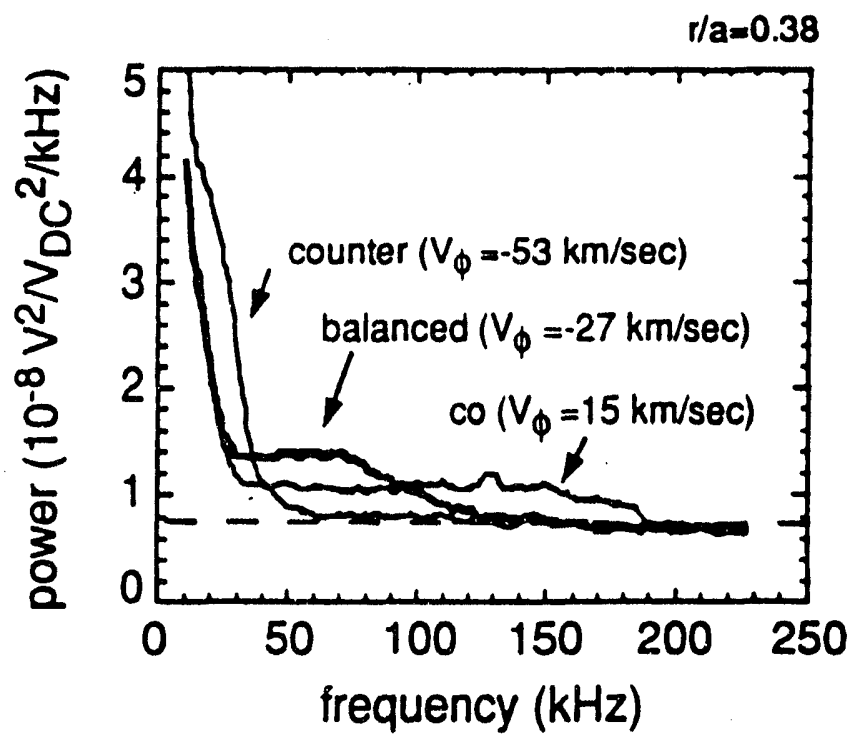

Figure 18. Observations of Doppler shifted autopower spectra. 


$$
\begin{array}{ll}
P_{\text {inj }}=9.6 \mathrm{MW}(\mathrm{bal}) & R=2.45 \mathrm{~m} \\
I_{p}=1.0 \mathrm{MA} & a=0.80 \mathrm{~m} \\
\text { Shots } 56346563485635156356 & q(a)=7.5
\end{array}
$$
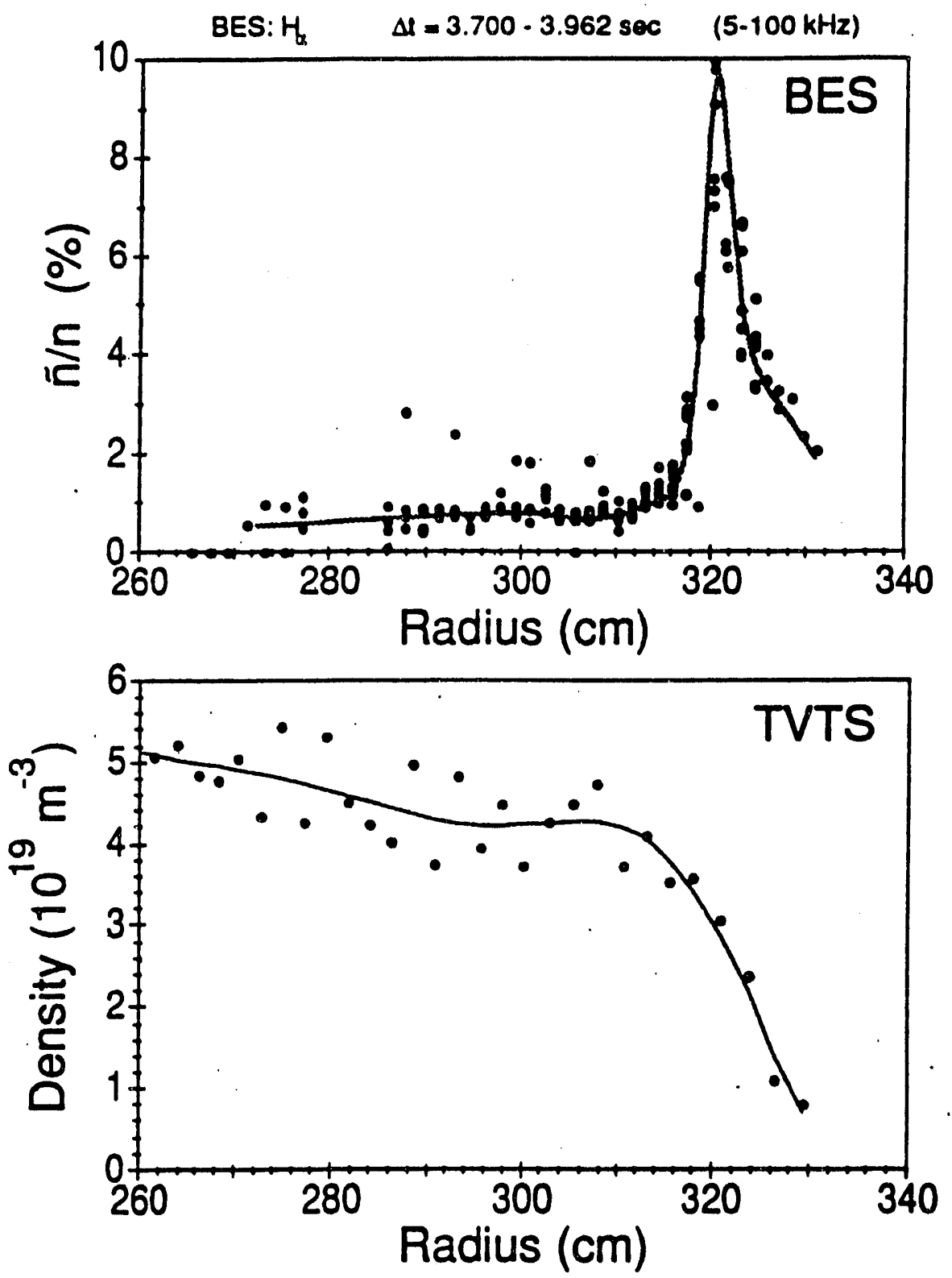

Figure 19. The density fluctuation amplitude, $\tilde{n}_{r m s} / n$, as a function of radius for typical L-mode discharge conditions. The fluctuations peak at the edge and decrease to less than $1 \%$ in the core (top). The profile of the electron density from Thomson scattering (bottom). 
TFTR L-mode Scan 11/2/90 Ip = IMA $\Delta t=3.700 \cdot 3.831 \mathrm{sec}$
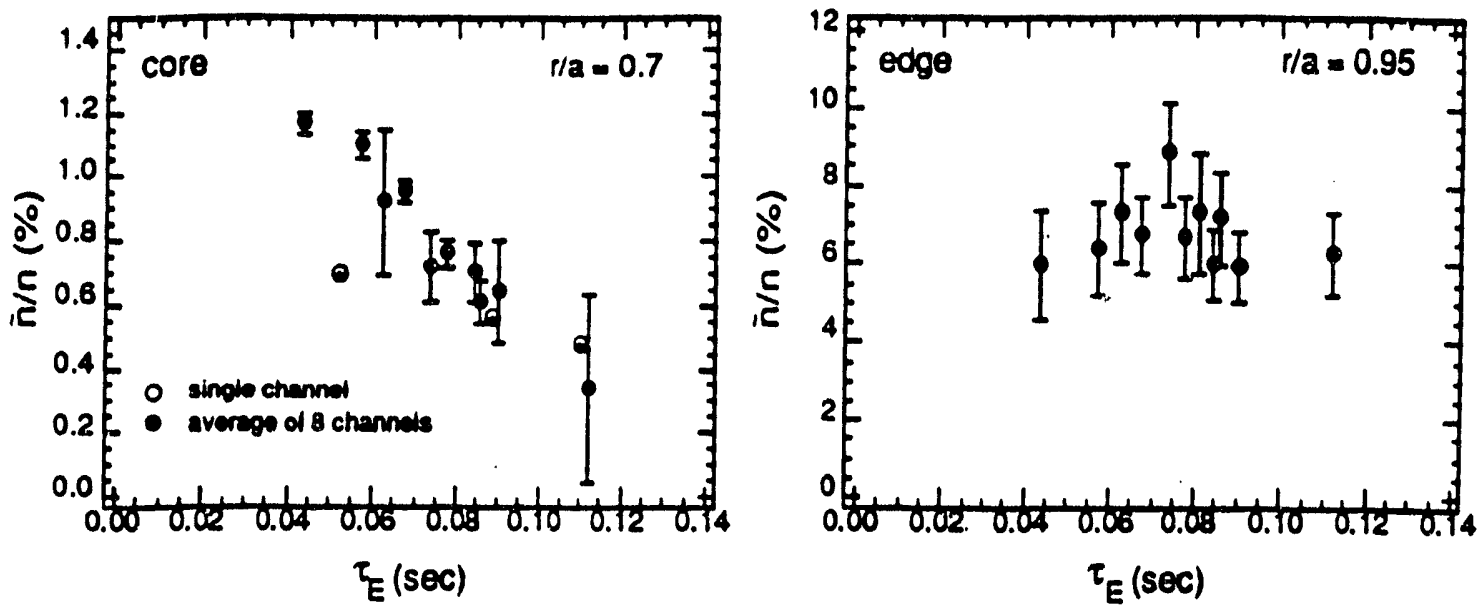

Figure 20. The fluctuation level as a function of confinement time in L-mode discharges. The fluctuations in the core scale with $\tau_{\mathrm{E}}$ while the edge fluctuations are independent of $\tau_{\mathrm{E}}$.

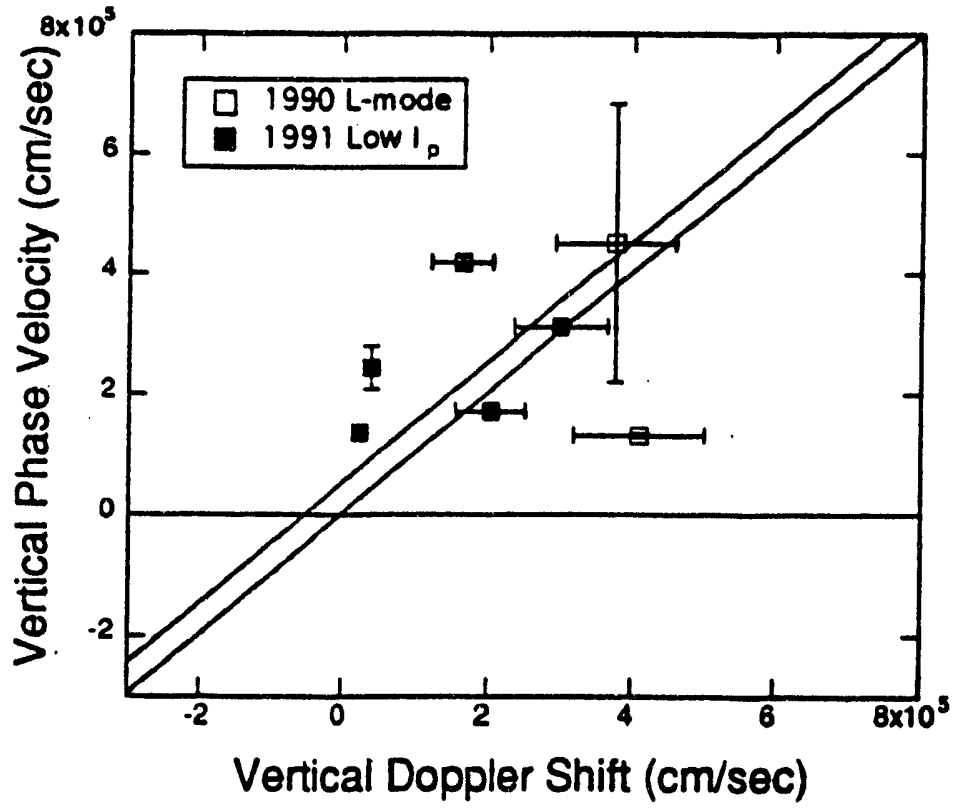

Figure 21 . The vertical phase velocity versus vertical doppler shift for standard L-mode and low-IP L-mode discharges. The 1991 data was acquired with the new PPD detectors and is thus of higher quality than the 1990 data. Except for one point from the 1990 run, the data are consistent with propagation in the ion diamagnetic direction. 

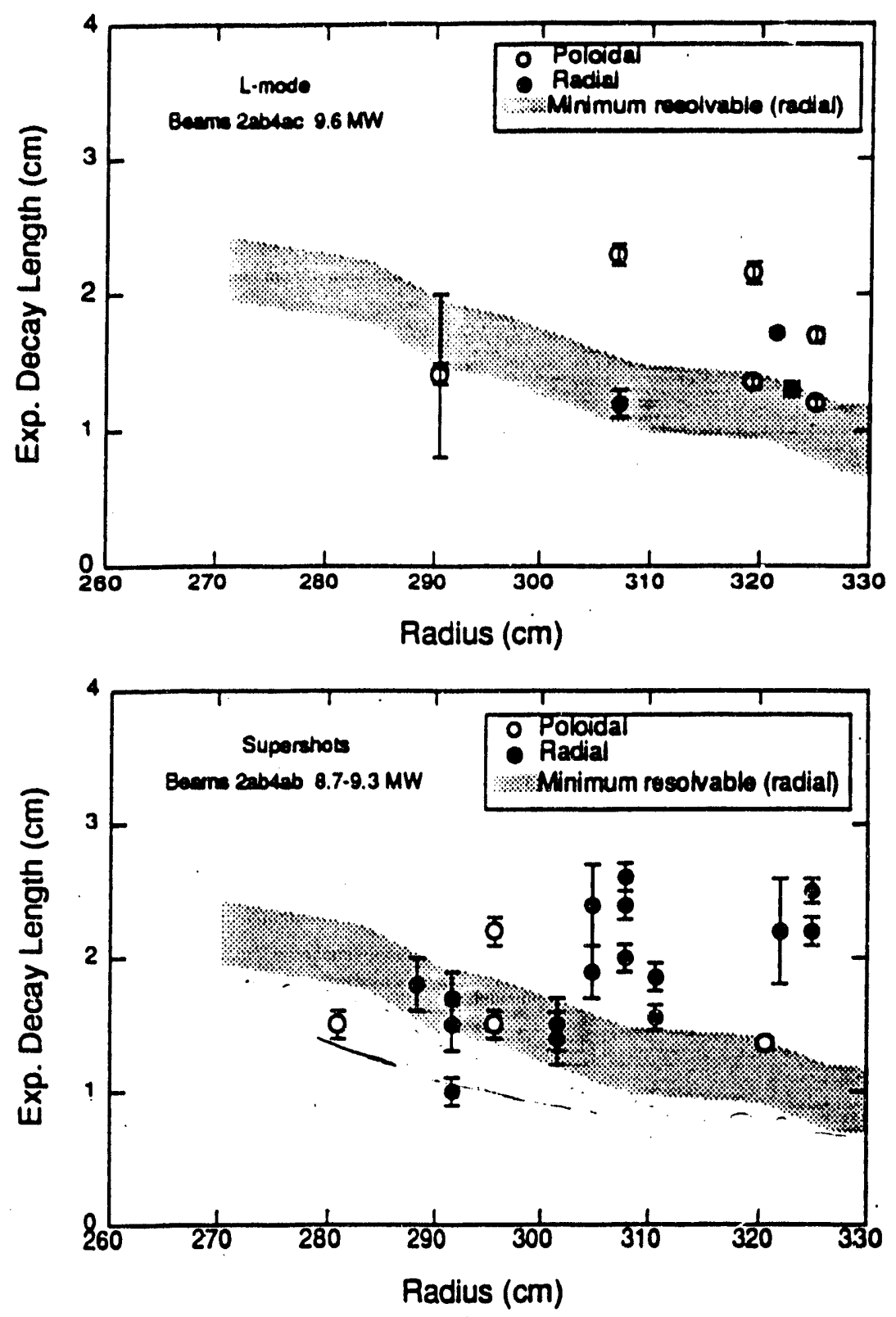

Figure 22. Correlation lengths as a function of radius for L-mode (top) and supershots (bottom). 


$$
\begin{array}{ll}
\text { BES: D-alpha } & \Delta t=3.900-4.400 \mathrm{sec} \\
\text { Shot } & \text { Pinj }=9.0 \mathrm{MW} \text { (bal) } \\
58940 & I p=0.8 \mathrm{MA} \\
58941 & R=2.45 \mathrm{~m} \\
58942 & a=0.80 \mathrm{~m} \\
58943 & q(a)=7.5
\end{array}
$$
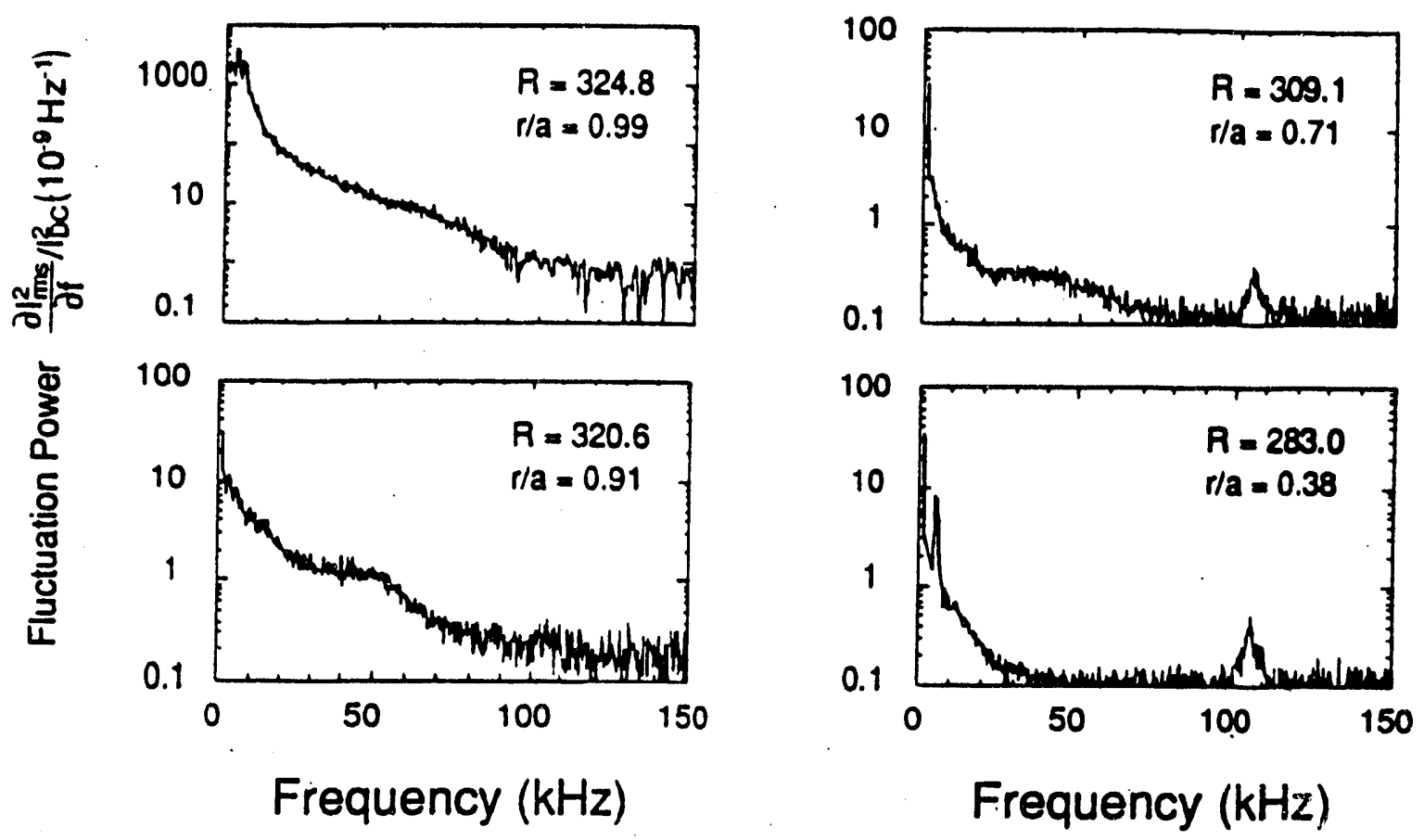

Figure 23. Autopower spectra from a typical supershot discharges. 


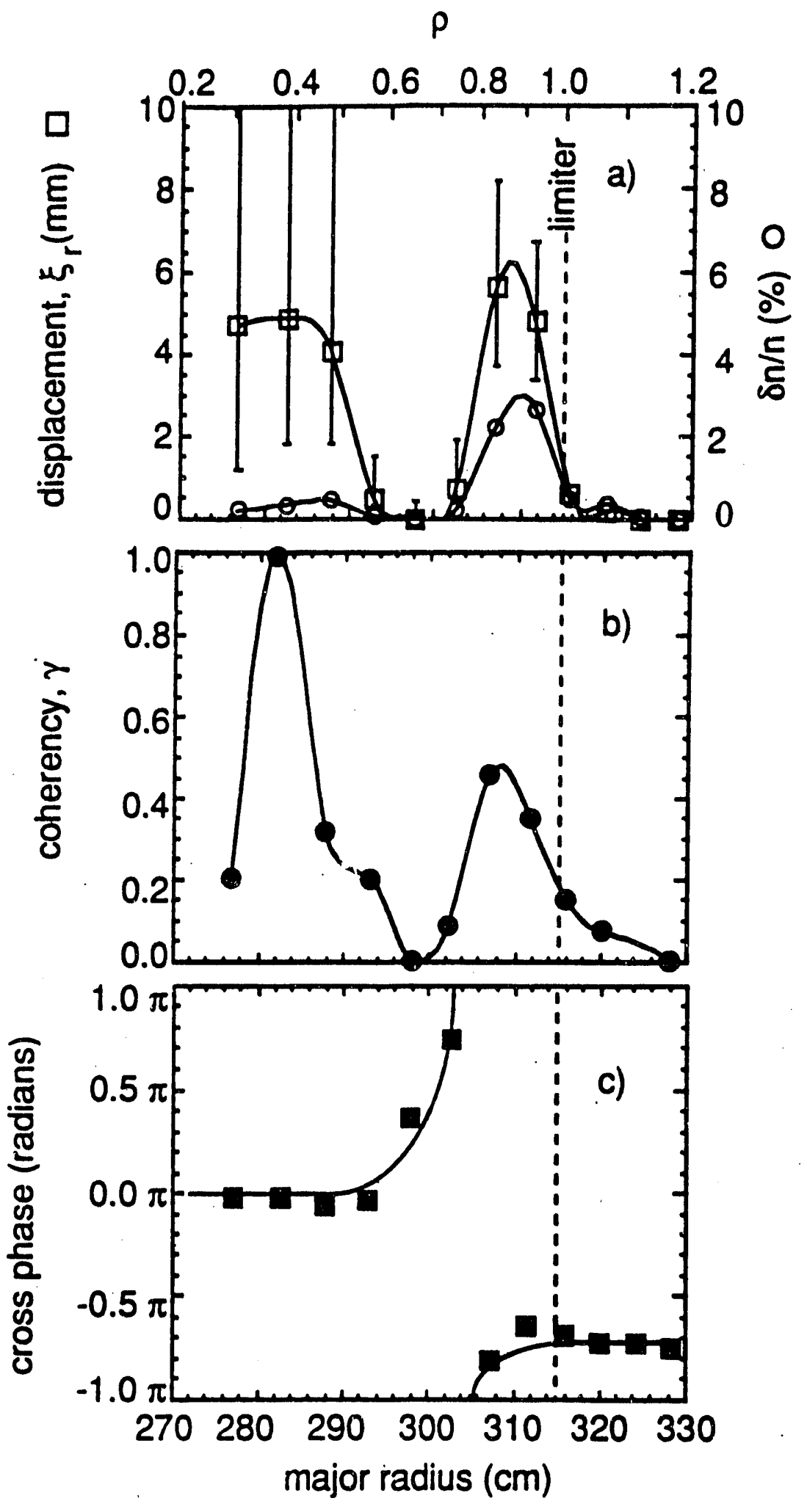

Figure 24. The radial structure of the Toroidal Alfvén Eigenmode in TFTR, amplitude and radial plasma displacement (a), coherency (b) and cross phase (c). 

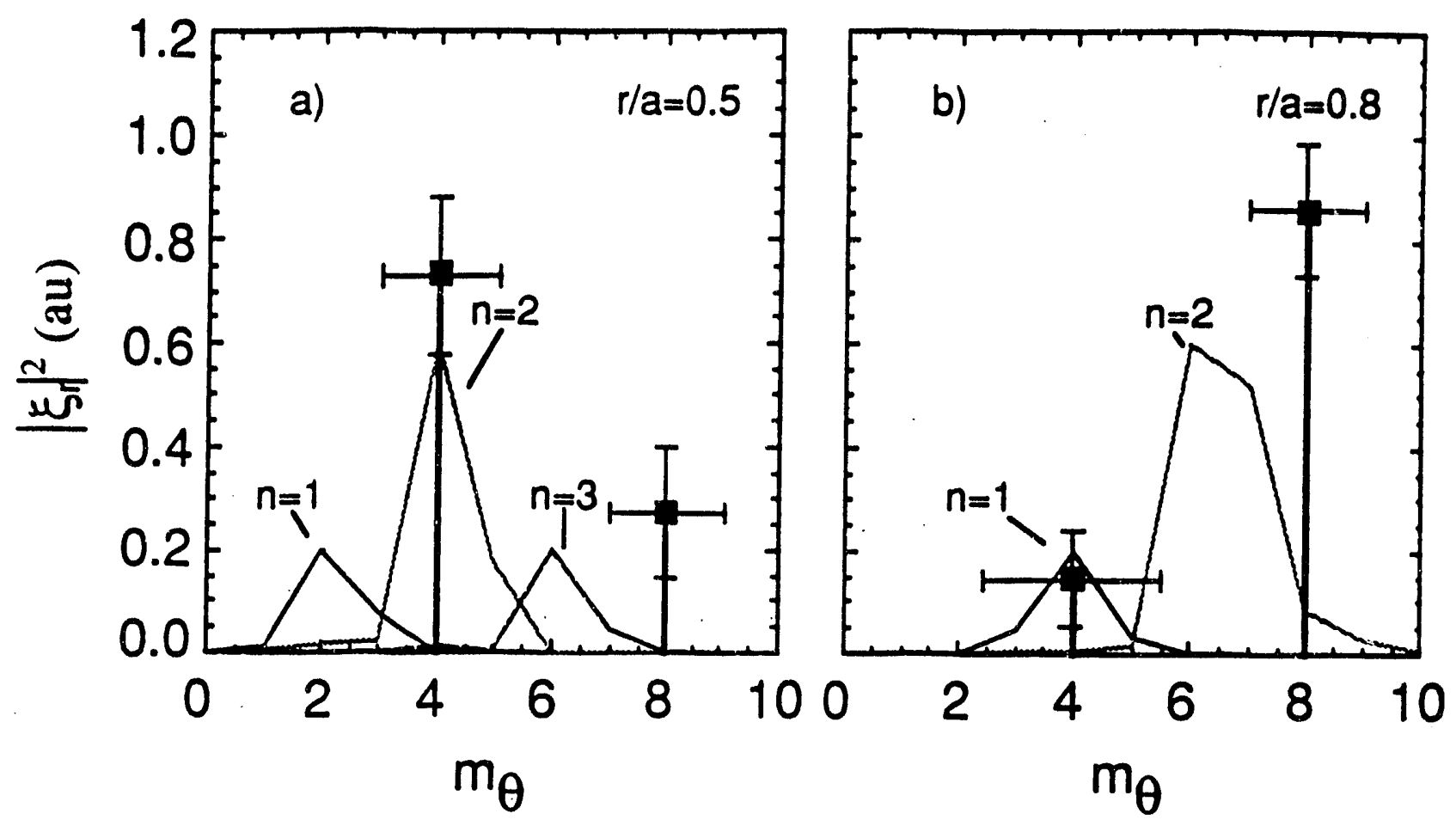

Figure 25. The poloidal mode spectrum of the TAE on TFTR at two radii. The labeled curves $(n=1,2,3)$ are simulated spectra from the resistive MHD code NOVA-K. 


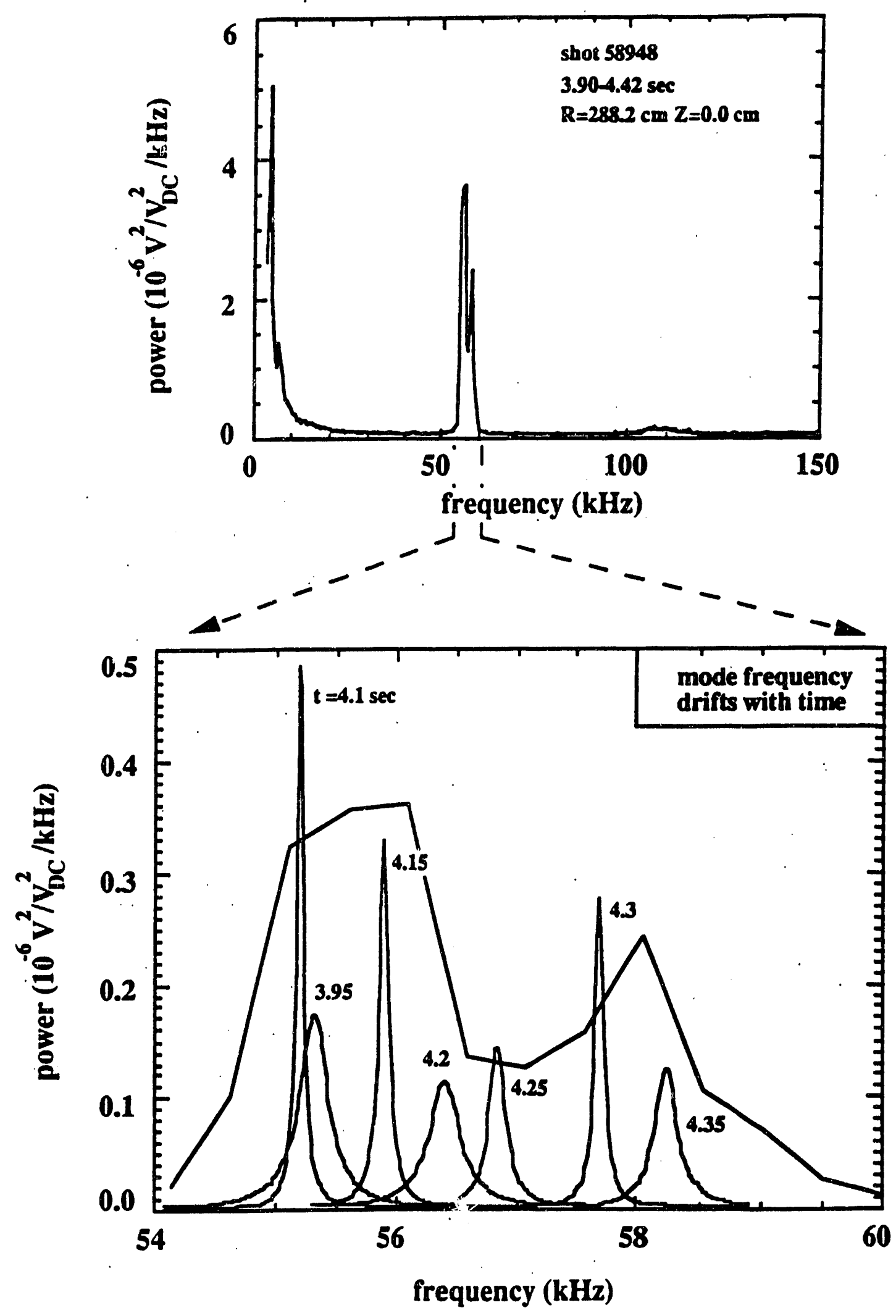

Figure 26. The autopower spectrum from a discharge with a large 4/3 magnetic island (near $57 \mathrm{kHz}$, above). The apparent spectral width of the mode is due to shifts in the move center frequency, as shown by the time-resolved spectra (below). 


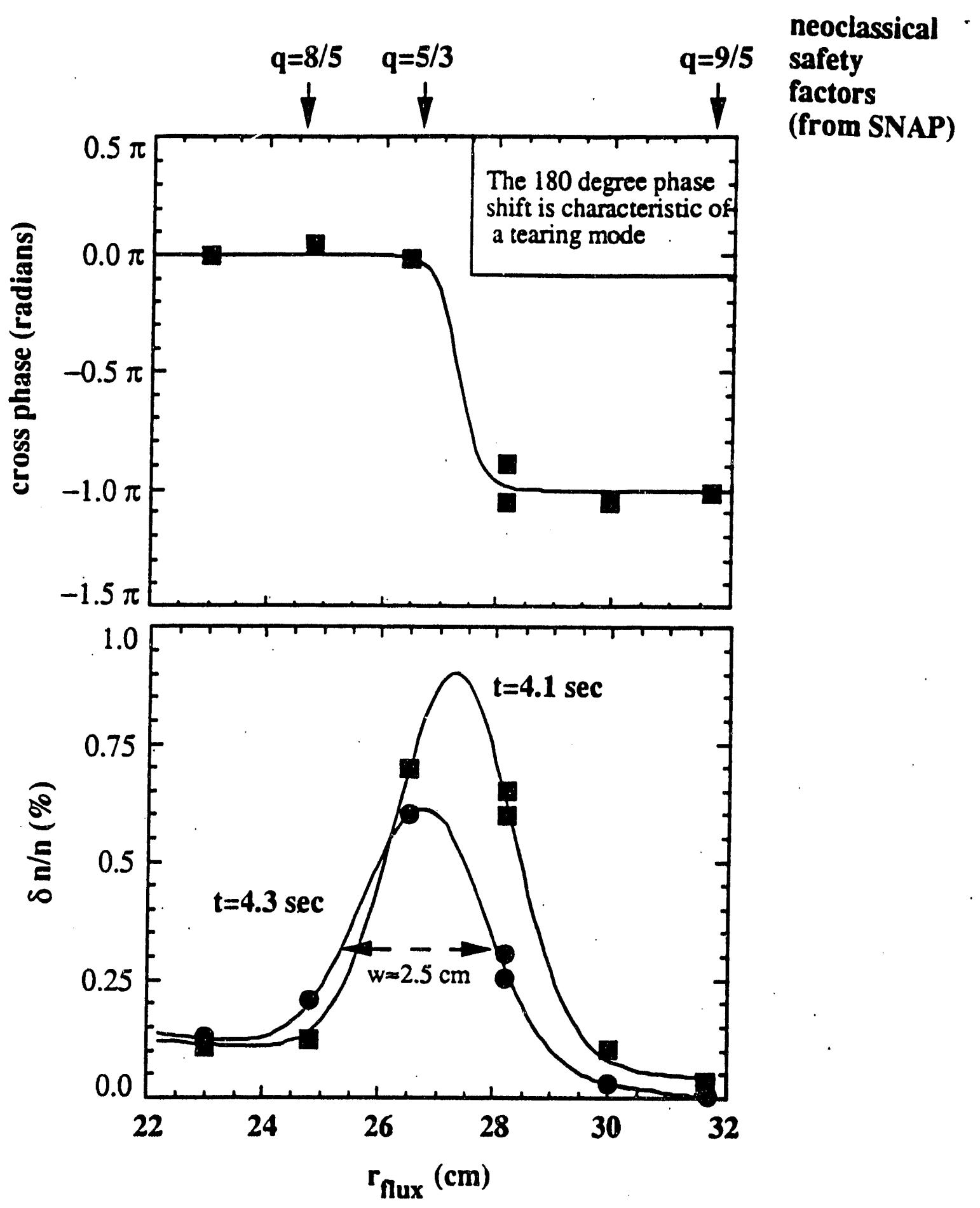

Figure 27. The radial structure of a $4 / 3$ tearing mode. 
High B-pol: No B collapse; Large Internal Island $\Rightarrow$ Flattening of Te profile

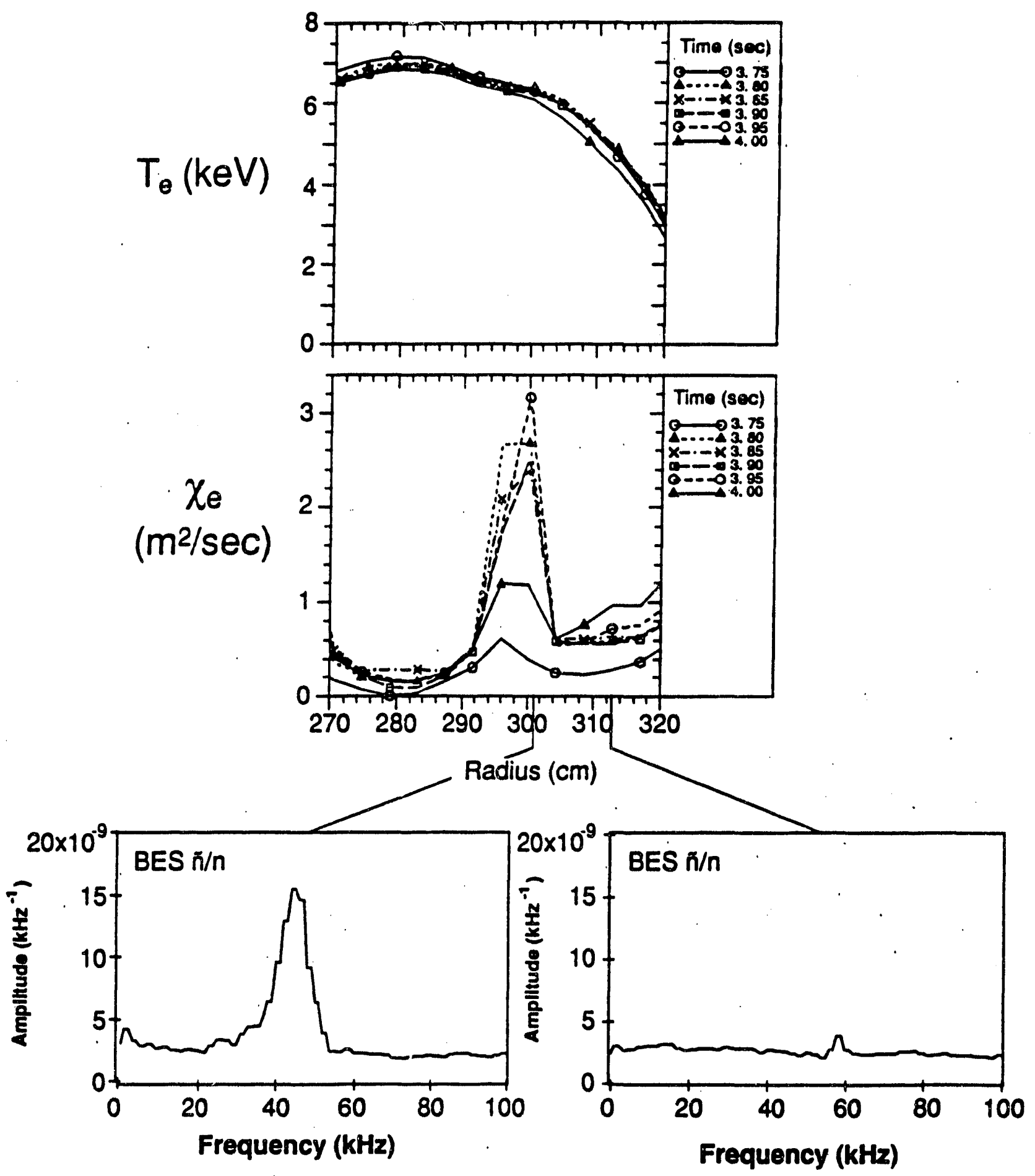

Figure 28. Observation of a large internal magnetic island near $300 \mathrm{~cm}$. The mode causes a local increase in the electron diffusivity. 


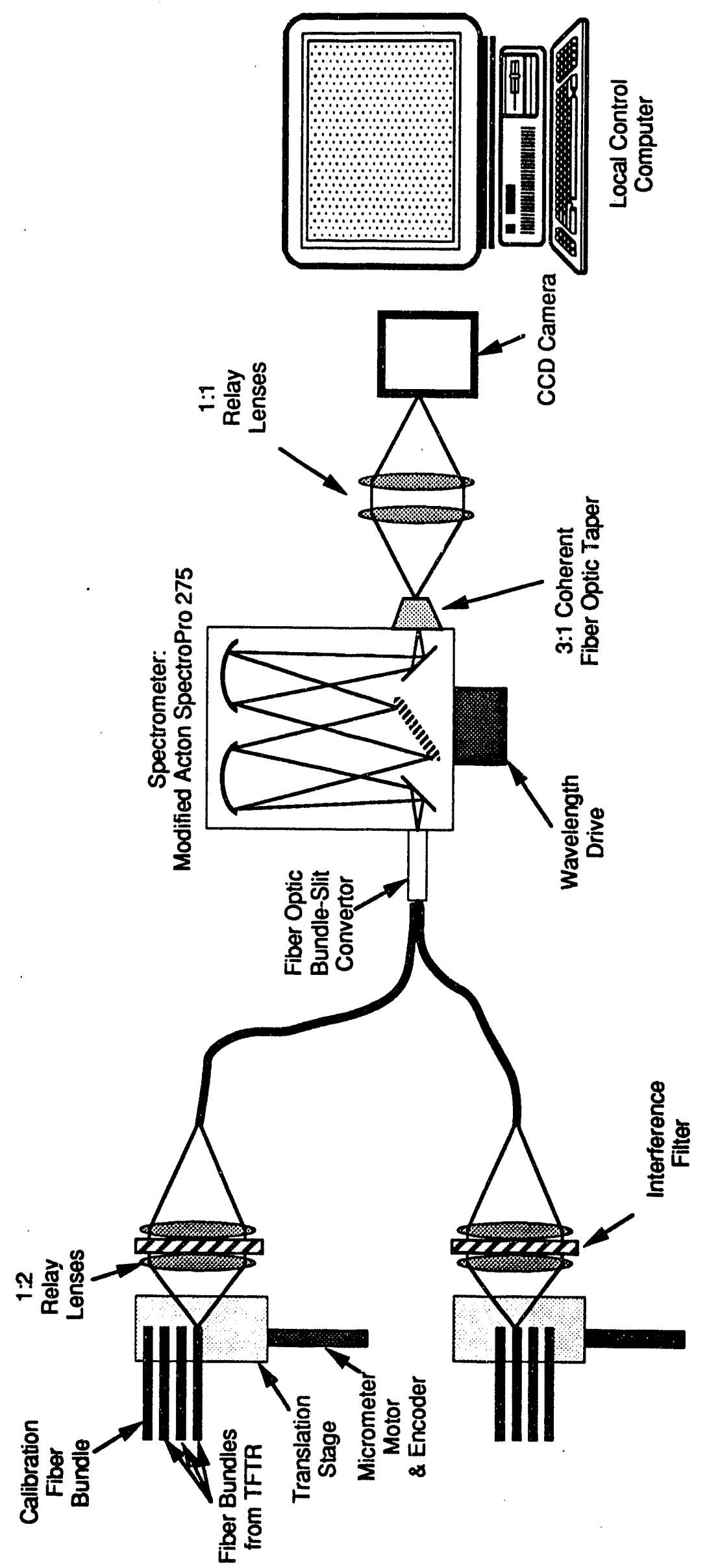

हี่ 

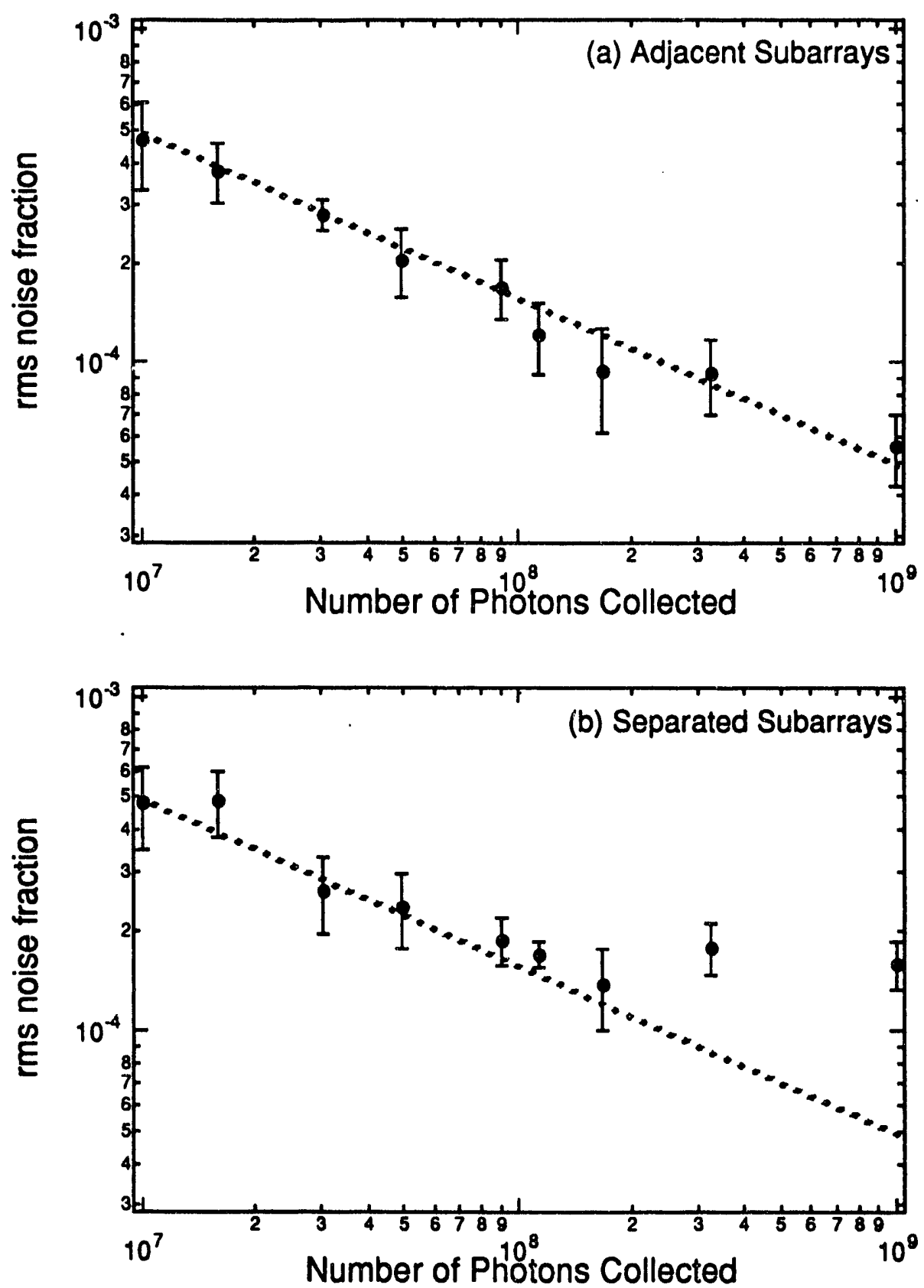

Fig. 30. Noise tests of CCD array for alpha-CHERS, showing measured rms noise as a function of number of photons collected in a subarray representing a spectral bin in the spectrometer focal plane. (a) Noise in relative response of two subarrays which lie adjacent to one another on the CCD chip. (b) Noise in relative response when the subarrays are physically separated from one another by a large fraction of the chip size. The dashed line indicates the value expected from pure photon statistics. The noise floor observed in (b) is thought to be due to variations in the local chip temperature. 

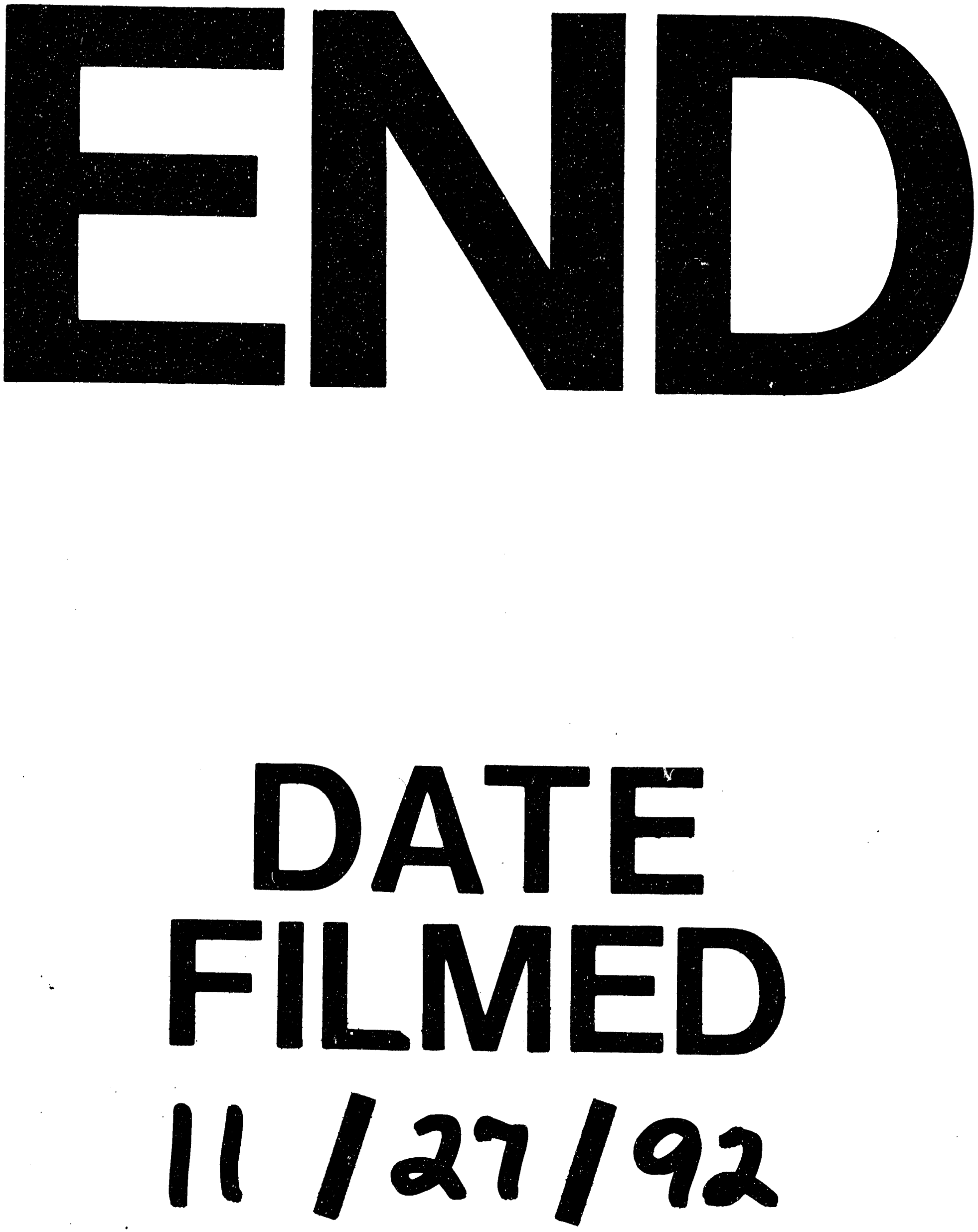
